\title{
Physics of selective conduction and point mutations in biological ion channels SUPPLEMENTAL MATERIAL
}

\author{
W.A.T. Gibby,${ }^{1, *}$ M.L. Barabash, ${ }^{1}$ C. Guardiani, ${ }^{1,2}$ D. G. Luchinsky, ${ }^{1,3, \dagger}$ and P.V.E. McClintock ${ }^{1, \ddagger}$ \\ ${ }^{1}$ Department of Physics, Lancaster University, Lancaster LA1 4YB, UK. \\ ${ }^{2}$ Department of Mechanical and Aerospace Engineering, Sapienza University, Rome, Italy \\ ${ }^{3}$ KBR, Inc., Ames Research Center, Moffett Field, CA, USA
}

(Dated: April 23, 2021)

\begin{abstract}
We elaborate on the theory developed in the main paper, for those readers who are interested in greater detail. We first discuss the statistical properties including the possible ion configurations in the pore and the link between our free energy and the potential of mean force (PMF). Then we derive the equations governing ionic conductivity at small applied potentials (linear response), and consider three important examples to further illuminate understanding the central figure of the main paper (Figure 3.). Finally we briefly review the important parameters $\Delta \bar{\mu}_{K, 1-4}$ and $\Delta \bar{\mu}_{N a, 1-4}$ and place them in the context of other results.
\end{abstract}

\section{CONTENTS}

Derivation of the statistical theory 1

Definition of the system 1

Configurations in the pore 3

Derivation of the ensemble and its properties 3

Derivation of the ionic conductivity 5

Electrostatics and effects of ionic binding 8

Effects of ion binding and polarisation 8

Electrostatic interaction 8

Novel insights from the theory 9

Fundamental importance of structure $\quad 9$

Principal results of the main paper 10

Proposed future work 11

Conductivity Examples 12

Example 1: One species, one ion, multiple binding sites 12

Example 2: One species, two ions, two binding sites 14

Example 3: Two species, multiple ions, multiple binding sites 14

Relation to PMF 15

Analysis of Parameters 16

References 17

DERIVATION OF THE STATISTICAL THEORY

Definition of the system

The system is described by Fig. 1 which is copied from the main text. It represents the selectivity filter (SF) of a biological channel. This pore (denoted by $(c)$ ) is thermally and diffusively coupled to the left $(L)$ and right $(R)$ bulk reservoirs $(b)$. Each bulk contains mixed solutions of $S$ total ionic species where $s \in 1, \cdots, S$. The system as a whole is characterized by the canonical ensemble with constant total particle number $N_{s}$, volume $V$ and temperature $T$. 


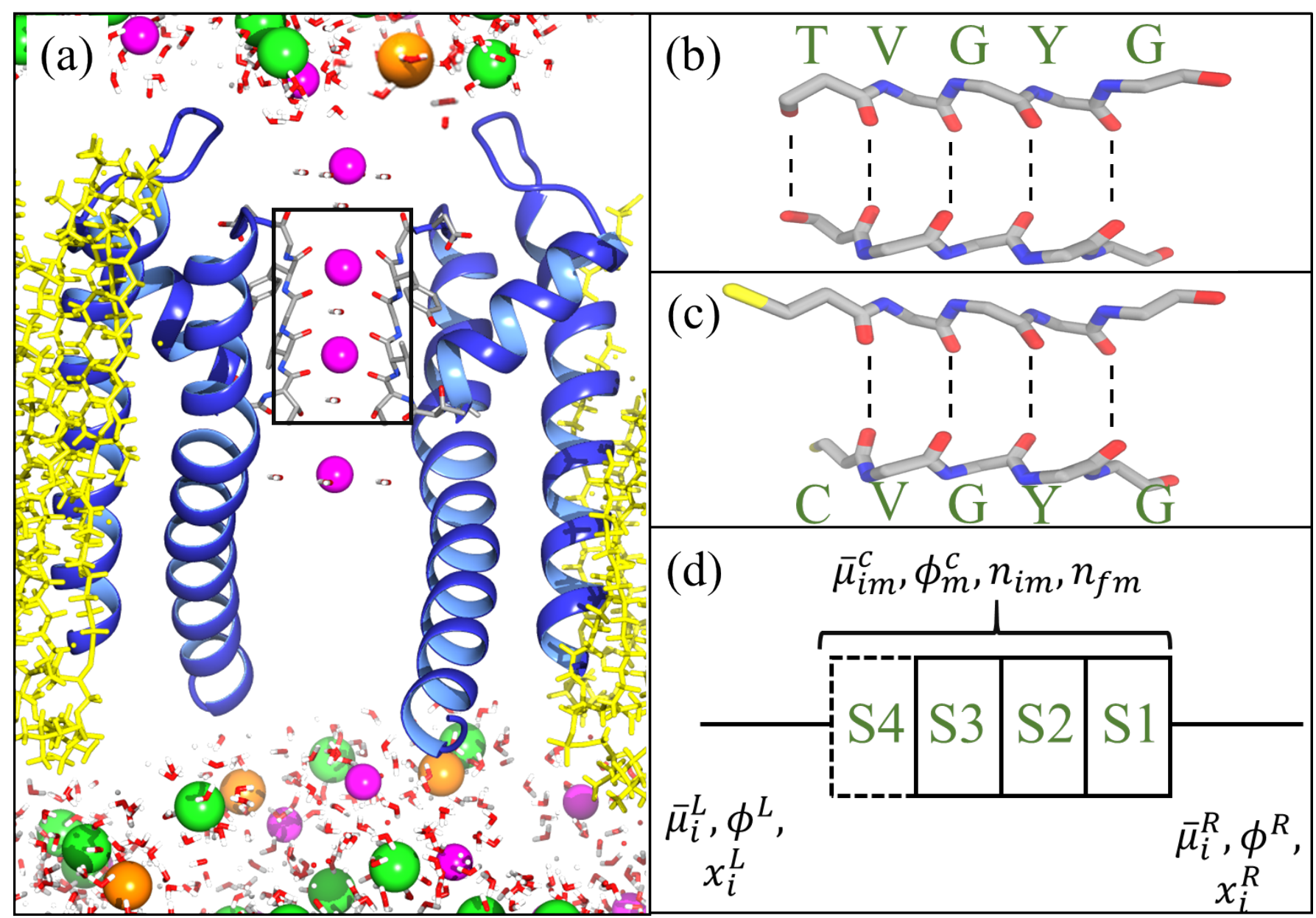

FIG. 1. Structure of open KcsA (5vk6.pdb) [2] visualised using chimera [3]. (a) Two chains (blue ribbons) spanning a lipid membrane (yellow strands) between two aqueous ionic solutions. The SF is located within the box, and $\mathrm{K}^{+}$(purple), $\mathrm{Na}^{+}$ (orange), and $\mathrm{Cl}^{-}$(green) ions alongside water molecules are included. (b) Structure of the SF for wild type KcsA and (c) the T75C mutant, with indicated amino acids at binding sites. (d) Lattice model used to define the system.

Ions are free to leave the bulk solution and bind at specific sites in the pore, with $n_{s}^{b}$ being the number of ions in either bulk. Due to the narrowness of the pore, ionic motion is confined to one dimensional conduction via a finite number of binding sites $M$, where each site $m \in 1, \cdots, M$ can hold a single ion at most. Therefore, the total number of ions in the pore is $n=\sum_{m}^{M} \sum_{s}^{S} n_{s m} \leq M$, where $\sum_{s=1}^{S} n_{s m} \in 0,1$ and $n_{s}=\sum_{m=1}^{M} n_{s m}$. All possible configurations of ionic binding in the pore $\left\{n_{s m}\right\}$ (or $\left\{n_{j}\right\}$ to simplify notations) are mutually exclusive leading to Fermi statistics [1]. As a result the total energy of each configuration in the system is found by explicitly counting the number of ions of each species that leave the left $\left(n_{s m}^{\prime \prime}\right)$ and right $\left(n_{s m}^{\prime}\right)$ bulks and enter site $m$ in the pore (keeping the total number of ions constant),

$$
E\left(\left\{n_{j}\right\}\right)=E_{0}+\sum_{s}^{S}\left(n_{s}^{L} \mu_{s}^{L}+n_{S}^{R} \mu_{s}^{R}\right)+\sum_{s}^{S} \sum_{m}^{M}\left(n_{s m}^{\prime}+n_{s m}^{\prime \prime}\right) \mu_{s m}^{c}
$$

Here we define $E_{0}=T S-p V$, to be the thermodynamic contribution to the energy of the whole system; with entropy $S$, pressure $p$. Terms: $\mu_{s}^{b}, \mu_{s m}^{c}$ correspond to the species electrochemical potentials in either bulk and site $m$ in the pore respectively. 


\section{Configurations in the pore}

The number of possible states corresponding to the total number of possible configurations $\left\{n_{j}\right\}$ of ions binding to the pore can be calculated explicitly. If we consider indistinguishable ions and empty sites (or holes) then we can use the multinomial theorem with each of the $i=1 \cdots S$ species plus empty sites $n_{0}$ contributing to the total number of states. We note that if the system is reduced to a single species then we can count the number of states using the Binomial theorem. Thus, for $n=\sum_{s} n_{s}$ ions in a pore with $M$ binding sites, the number of states can be calculated from,

$$
\mathcal{M}\left(M, n_{1}, \cdots n_{S}, n_{0}\right)=\left(\begin{array}{c}
M \\
n_{1}, \cdots, n_{S}, n_{0}
\end{array}\right)=\frac{M !}{n_{0} ! \prod_{s} n_{s} !},
$$

where $n_{0}=M-\sum_{s} n_{s}$. It is clear that Eq. (2) already accounts for the indistinguishability of ions and is the reason why the $k T \log \left(n_{s}\right)$ ! term is included in the expression for the total energy (see (1) of the main text). Therefore the total number of states $\mathcal{N}$ is given by the sum of this coefficient over all possible numbers of ions and configurations of species inside the pore. Hence, $\mathcal{N}$ is given by the sum over all possible values of $n_{s}$ and $n_{0}$ that satisfy the condition $n_{0}+\sum_{s} n_{s}=M$,

$$
\mathcal{N}=\sum_{n_{0}+\sum_{s} n_{s}=M} \mathcal{M}\left(M, n_{1}, \cdots n_{S}, n_{0}\right)
$$

There are four binding sites in the selectivity filter of $\operatorname{KcsA}(M=4)[4]$ and so, if we consider a single species of permeant ions, then we find 15 possible states. This number rises to 65 if we consider two conducting species.

\section{Derivation of the ensemble and its properties}

The electrochemical potential in the bulk takes the standard form defined [5-7] as the sum of ideal, excess, and electrostatic parts

$$
\mu_{s}^{b}=k T \ln \left(\frac{\left(\Lambda_{s}^{b}\right)^{3} x_{s}^{b}}{q_{s}^{\text {int }, b}}\right)+\bar{\mu}_{s}^{b}+q z_{s} \phi^{b}
$$

where $\Lambda_{s}^{b}, q_{s}^{\mathrm{int}, b}, x_{s}^{b}, z_{s}, \phi^{b}$ and $\bar{\mu}_{s}^{b}$ denote the thermal wavelength, internal partition function, mole fraction, valence, external electric and excess chemical potential respectively.

Since the number of ions inside the pore is less than $M$ and small typically $M \sim \mathcal{O}(1)$, the energy to add an ion to site $m$, can be defined from the discrete difference in the energy of the pore between $n$ and $n+1$ states [8]

$$
\mu_{s m}^{c}\left(\left\{n_{j}\right\}\right)=k T \ln \frac{\left(\Lambda_{s}^{c}\right)^{3} \Delta f\left(\left\{n_{j}\right\}\right)}{q_{s}^{\text {int }, c}}+\bar{\mu}_{s m}^{c}+q z_{s} \phi_{m}^{c}+\Delta \mathcal{E} .
$$

In this expression $\Lambda_{s}^{c}, q_{s}^{\mathrm{int}, c}, \phi_{m}^{c}$ and $\bar{\mu}_{s m}^{c}$ are as defined previously except for the pore and site $m$. Since we assume that: $\left(\Lambda_{s}^{b}\right)^{3} / q_{s}^{\text {int }, b} \sim\left(\Lambda_{s}^{c} s\right)^{3} / q_{s}^{\text {int }, c}$, we can cancel these terms in the energy expression and so shall ignore their contribution in all future expressions. The remaining two terms $\Delta f\left(\left\{n_{j}\right\}\right)$ and $\Delta \mathcal{E}\left(\left\{n_{j}\right\}\right)$ are the differences in number of ions in the pore and electrostatic energy respectively. Thus $\Delta f$ is related to the indistinguishably of ions in the pore

$$
\Delta f\left\{n_{j}\right\}=k T \log \left(\frac{n_{s}+1}{n_{0}-1}\right)
$$

where we recall that $n_{0}$ is the number of 'holes' or empty sites $n_{0}=M-\sum_{s}^{S} n_{s}$. Clearly these two terms depend on the initial state of the system before adding an ion. As a result the energy corresponding to ions inside the pore can be expressed as follows,

$$
\begin{aligned}
\sum_{s}^{S} \sum_{m}^{M}\left(n_{s m}^{\prime}+n_{s m}^{\prime \prime}\right) \mu_{s m}^{c}\left(\left\{n_{j}\right\}\right) & =\sum_{s}^{S} \sum_{m}^{M}\left(n_{s m}^{\prime}+n_{s m}^{\prime \prime}\right)\left[\bar{\mu}_{s m}^{c}+q z_{s} \phi_{m}^{c}\right] \\
& +f\left(\left\{n_{j}\right\}\right)+\mathcal{E}\left(\left\{n_{j}\right\}\right)-\mathcal{E}(0)-f(0)
\end{aligned}
$$


where $\mathcal{E}(0)$ and $f(0)$ denote the electrostatic energy and factor $f$ to have zero ions in the pore. These two terms are constant, and will be factored out of the expression and so we shall neglect their presence hereafter.

With these terms in mind, we can now rewrite the total energy Eqn. (1) by introducing $N_{s}^{b}$ defined as the number of ions in either bulk if the pore is empty i.e.,

$$
N_{s}^{L}=n_{s}^{L}+\sum_{m}^{M} n_{s m}^{\prime \prime}
$$

and similarly for $N_{s}^{R}$. Thus, the energy becomes

$$
E\left(\left\{n_{j}\right\}\right)=E_{0}+\sum_{s}^{S}\left[\left(N_{s}^{L}-n_{s m}^{\prime \prime}\right) \mu_{s}^{L}+\left(N_{S}^{R}-n_{s m}^{\prime}\right) \mu_{s}^{R}\right]+\sum_{m}^{M}\left(n_{s m}^{\prime}+n_{s m}^{\prime \prime}\right) \mu_{m}^{c}
$$

which can be written as,

$$
\begin{aligned}
& E\left(\left\{n_{j}\right\}\right)=E_{0}+\mathcal{E}\left(\left\{n_{j}\right\}\right)+\sum_{s=1}^{S}\left(N_{s}^{L}-\sum_{m=1}^{M} n_{s m}^{\prime \prime}\right) \mu_{s}^{L}+\sum_{s=1}\left(N_{s}^{R}-\sum_{m=1}^{M} n_{s m}^{\prime}\right) \mu_{s}^{R}+k T \ln \left(n_{0}\right) !+\sum_{s=1}^{S} k T \ln n_{s} ! \\
& +\sum_{s=1}^{S} \sum_{m=1}^{M}\left(n_{s m}^{\prime}+n_{s m}^{\prime \prime}\right)\left(\bar{\mu}_{s m}^{c}+q z_{s} \phi_{m}^{c}\right),
\end{aligned}
$$

where we have introduced $f=\sum_{s} k T \log \left(n_{s} !\right)+k T \log \left(n_{0} !\right)$. If we now collect terms proportional to $N_{s}^{b}$, and because at equilibrium, $\mu_{s}^{L}=\mu_{s}^{R}=\mu_{s}^{b}$, we can write,

$$
\begin{aligned}
& E\left(\left\{n_{j}\right\}\right)=E_{0}+\mathcal{E}\left(\left\{n_{j}\right\}\right)+\sum_{s=1}^{S}\left(N_{s}^{L}+N_{s}^{R}-\sum_{m=1}^{M}\left(n_{s m}^{\prime \prime}+n_{s m}^{\prime}\right)\right) \mu_{s}^{b}+k T \ln \left(n_{0}\right) !+\sum_{s=1}^{S} k T \ln n_{s} ! \\
& +\sum_{s=1}^{S} \sum_{m=1}^{M}\left(n_{s m}^{\prime}+n_{s m}^{\prime \prime}\right)\left(\bar{\mu}_{s m}^{c}+q z_{s} \phi_{m}^{c}\right) .
\end{aligned}
$$

We recall that total number of particles is defined by: $N_{s}=N_{s}^{L}+N_{s}^{R}$, and hence, we can rewrite $N_{s} \mu_{s}^{b}$ as a constant. Constant terms can be factored out of the total energy, and thus we arrive at the total energy of the system,

$$
\begin{aligned}
& E\left(\left\{n_{j}\right\}\right)=E_{0}+\mathcal{E}\left(\left\{n_{j}\right\}\right)+k T \ln \left(n_{0}\right) !+\sum_{s=1}^{S} k T \ln n_{s} ! \\
& +\sum_{s=1}^{S} \sum_{m=1}^{M}\left(n_{s m}\right)\left(\bar{\mu}_{s m}^{c}+q z_{s} \phi_{m}^{c}-\mu_{s}^{b}\right),
\end{aligned}
$$

where we have reintroduced the number of ions in each site $m$ of the pore, $n_{s m}=n_{s m}^{\prime}+n_{s m}^{\prime \prime}$. The stationary equilibrium probability-distribution function $P\left(\left\{n_{j}\right\}\right)$ for particular occupation configurations is given by the Gibbs distribution,

$$
P\left(\left\{n_{j}\right\}\right)=e^{-E\left(\left\{n_{j}\right\}\right) / k T} Z^{-1} .
$$

However, we note that there is explicitly a constant term in $E\left(\left\{n_{j}\right\}\right)$ and because we shall assume that the thermodynamic contribution $E_{0}$ does not depend on the state of the system, we can factor this term and write the effective grand canonical ensemble (GCE) distribution function as,

$$
P\left(\left\{n_{j}\right\}\right)=\frac{1}{n_{0} ! \prod_{s}^{S} n_{s} !} e^{-\left(\mathcal{E}\left(\left\{n_{j}\right\}\right)+\sum_{s=1}^{S} \sum_{m=1}^{M}\left(n_{s m}\right)\left(\bar{\mu}_{s m}^{c}+q z_{s} \phi_{m}^{c}-\mu_{s}^{b}\right)\right) / k T} Z^{-1} .
$$

The partition function $Z$ takes the form,

$$
Z=\sum_{\left\{n_{j}\right\}} \frac{1}{n_{0} ! \prod_{s}^{S} n_{s} !} e^{-\left(\mathcal{E}\left(\left\{n_{j}\right\}\right)+\sum_{s=1}^{S} \sum_{m=1}^{M}\left(n_{s m}\right)\left(\bar{\mu}_{s m}^{c}+q z_{s} \phi_{m}^{c}-\mu_{s}^{b}\right)\right) / k T} .
$$

Finally the Gibb's free energy $(\mathrm{G}=\mathrm{E}-\mathrm{TS}+\mathrm{pV})$ can be written as,

$$
G\left(\left\{n_{j}\right\}\right)=\mathcal{E}\left(\left\{n_{j}\right\}\right)+k T \ln \left(n_{0}\right) !+\sum_{s=1}^{S} k T \ln n_{s} !+\sum_{s=1}^{S} \sum_{m=1}^{M}\left(n_{s m}\right)\left(\bar{\mu}_{s m}^{c}+q z_{s} \phi_{m}^{c}-\mu_{s}^{b}\right) .
$$




\section{DERIVATION OF THE IONIC CONDUCTIVITY}

In this section, we aim to derive the conduction properties of our system in the linear response limit. We consider a small applied electric potential held constant for a long time, and follow Kubo and Zwanzig to investigate the static response using equilibrium statistical mechanics [9-11]. Thus we start by perturbing the total energy of the system Eqn. (10) by a static potential $\delta \phi$, and since the system stays in chemical equilibrium it is defined by $\delta \phi=\phi^{L}-\phi^{R}=\mu^{L}-\mu^{R}$. We shall perturb $\phi^{b}$ symmetrically by $\pm \delta \phi / 2$. The potential at each site in the pore $\phi_{m}^{c}$, is defined by $\alpha_{m} \delta \phi$, where $\alpha_{m}$ is the distance between the left bulk and each binding site. Hence we can write,

$$
\begin{aligned}
& E\left(\left\{n_{j}\right\}, \delta \phi\right)=E_{0}+\mathcal{E}\left(\left\{n_{j}\right\}\right)+\sum_{s=1}^{S}\left(N_{s}^{L}-\sum_{m=1}^{M} n_{s m}^{\prime \prime}\right)\left(+q z_{s} \delta \phi / 2+\bar{\mu}_{s}^{L}+k T \log \left(x_{s}^{L}\right)\right) \\
& +\sum_{s=1}\left(N_{s}^{R}-\sum_{m=1}^{M} n_{s m}^{\prime}\right)\left(-q z_{s} \delta \phi / 2+\bar{\mu}_{s}^{R}+k T \log \left(x_{s}^{R}\right)\right) \\
& +k T \ln \left(n_{0}\right) !+\sum_{s=1}^{S} k T \ln n_{s} !+\sum_{s=1}^{S} \sum_{m=1}^{M}\left(n_{s m}^{\prime}+n_{s m}^{\prime \prime}\right)\left(\bar{\mu}_{s m}^{c}+q z_{s} \alpha_{m} \delta \phi\right),
\end{aligned}
$$

Since the only difference between bulks is the electrostatic potential, we can simplify notation by writing $\mu_{s}^{0}=$ $\bar{\mu}_{s}^{b}+k T \log \left(x_{s}^{b}\right)$. Hence we by collecting terms with respect to $\delta \phi$ we can write

$$
\begin{aligned}
& E\left(\left\{n_{j}\right\}, \delta \phi\right)=E_{0}+\mathcal{E}\left(\left\{n_{j}\right\}\right) \\
& +\sum_{s=1}^{S}\left(N_{s}^{L}-\sum_{m=1}^{M} n_{s m}^{\prime \prime}\right)\left(+q z_{s} \delta \phi / 2\right)+\sum_{s=1}\left(N_{s}^{R}-\sum_{m=1}^{M} n_{s m}^{\prime}\right)\left(-q z_{s} \delta \phi / 2\right)+\sum_{s=1}^{S} \sum_{m=1}^{M}\left(n_{s m}^{\prime}+n_{s m}^{\prime \prime}\right) q z_{s} \alpha_{m} \delta \phi \\
& +\sum_{s=1}^{S}\left(N_{s}^{L}+N_{s}^{R}-\sum_{m=1}^{M}\left(n_{s m}^{\prime \prime}+n_{s m}^{\prime}\right)\right)\left(\bar{\mu}_{s}^{0}+k T \log \left(x_{s}^{0}\right)\right)+k T \ln \left(n_{0}\right) !+\sum_{s=1}^{S} k T \ln n_{s} !+\sum_{s=1}^{S} \sum_{m=1}^{M}\left(n_{s m}^{\prime}+n_{s m}^{\prime \prime}\right) \bar{\mu}_{s m}^{c},
\end{aligned}
$$

since in this instance the bulks are identical apart from the electrical potential: $N_{s}^{L}=N_{s}^{R}$ and so we can simplify the terms to write,

$$
\begin{aligned}
& E\left(\left\{n_{j}\right\}, \delta \phi\right)=E_{0}+\mathcal{E}\left(\left\{n_{j}\right\}\right) \\
& +\sum_{s=1}^{S}\left(\sum_{m=1}^{M}\left(n_{s m}^{\prime}-n_{s m}^{\prime \prime}\right)\right)\left(q z_{s} \delta \phi / 2\right)+\sum_{s=1}^{S} \sum_{m=1}^{M}\left(n_{s m}^{\prime}+n_{s m}^{\prime \prime}\right) q z_{s} \alpha_{m} \delta \phi \\
& +\sum_{s=1}^{S}\left(N_{s}^{L}+N_{s}^{R}-\sum_{m=1}^{M}\left(n_{s m}^{\prime \prime}+n_{s m}^{\prime}\right)\right)\left(\bar{\mu}_{s}^{0}+k T \log \left(x_{s}^{0}\right)\right)+k T \ln \left(n_{0}\right) !+\sum_{s=1}^{S} k T \ln n_{s} !+\sum_{s=1}^{S} \sum_{m=1}^{M}\left(n_{s m}^{\prime}+n_{s m}^{\prime \prime}\right) \bar{\mu}_{s m}^{c},
\end{aligned}
$$

We shall now introduce the parameter $v_{s m}^{b}$. This is defined for an ion entering the pore from the left and right bulk respectively and for each species separately $\left[1 / 2-\alpha_{m}, \alpha_{m}+1 / 2\right]$. We can write the following free energy, distribution and partition functions,

$$
\begin{aligned}
& G\left(\left\{n_{j}\right\} ; n_{f} ; \phi\right)=\mathcal{E}\left(\left\{n_{j}\right\}\right)+k T \sum_{s} \ln n_{s} !+k T \ln n_{0} !-\sum_{s} \sum_{m} n_{s m}\left[\Delta \bar{\mu}_{s m}+k T \ln \left(x_{s}\right) \pm q z_{s} v_{s m}^{b} \delta \phi\right] \\
& P\left(\left\{n_{j}\right\} ; n_{f} ; \phi\right)=\mathcal{Z}^{-1}\left(\frac{1}{n_{0} !} \prod_{s} \frac{\left(x_{s}^{b}\right)^{n_{s}}}{n_{s} !}\right) \times \exp \left[\left(\sum_{s=1}^{S} \sum_{m=1}^{M} n_{s m}\left(\Delta \bar{\mu}_{s m}^{b} \pm q z_{s} v_{s m}^{b} \delta \phi\right)-\mathcal{E}\left(\left\{n_{j}\right\} ; n_{f}\right)\right) / k T\right] \\
& Z\left(\left\{n_{j}\right\} ; n_{f} ; \phi\right)=\sum_{\left\{n_{j}\right\}}\left(\frac{1}{n_{0} !} \prod_{s} \frac{\left(x_{s}^{b}\right)^{n_{s}}}{n_{s} !}\right) \times \exp \left[\left(\sum_{s=1}^{S} \sum_{m=1}^{M} n_{s m}\left(\Delta \bar{\mu}_{s m}^{b} \pm q z_{s} v_{s m}^{b} \delta \phi\right)-\mathcal{E}\left(\left\{n_{j}\right\} ; n_{f}\right)\right) / k T\right] .
\end{aligned}
$$

In the limit of small $\delta \phi$ these expressions can be linearised,

$$
Z(\delta \phi) \approx Z\left[1 \pm \frac{q \delta \phi}{k T}\left\langle\sum_{s} \sum_{m} z_{s} n_{s m} v_{s m}^{b}\right\rangle\right]
$$


and,

$$
P(\delta \phi) \approx P\left[1 \pm \frac{q \delta \phi}{k T}\left(\sum_{s} \sum_{m} z_{s} n_{s m} v_{s m}^{b}-\left\langle\sum_{s} \sum_{m} z_{s} n_{s m} v_{s m}^{b}\right\rangle\right)\right]
$$

These expressions can be further simplified because parameter $v_{s m}^{b}$ can be averaged for each species around all sites in the pore: $\sum_{m} n_{s m} v_{s m}^{b}=n_{s} \hat{v}_{s}^{b}$. We note that if the pore has evenly distributed sites i.e. i.e. a symmetrical pore, then $\hat{v}_{s}^{b}=1 / 2$. In this publication we only consider a symmetrical pore, and the effects of varying this parameter will be investigated in future work.

To linear order the average of any dynamical variable $A$ in an applied field $E$ can be written in terms of the static susceptibility $\chi_{A M}[9]$,

$$
\langle A\rangle_{E}=\langle A\rangle+\chi_{A M} E .
$$

The response or $\chi_{A M}$ describes the average linear response produced by the applied field, and contains information about the coupling $M$ to the field.

We shall calculate the static susceptibility for the whole pore and the binding site $m$, in response to the field generated by the scalar potential $\delta \phi$. As a result, in accordance with Kubo, we shall be looking for a relation of the form,

$$
\langle n\rangle_{\phi}=\langle n\rangle+q \chi \delta \phi .
$$

where $\chi$ has units of $\left[k T^{-1}\right]$ or $\left[\left(m^{3} k T\right)^{-1}\right]$ depending on if it is the response for particle number or density.

If we first consider the the full pore, then we must average over total particle number inside the pore $\left\langle n_{s}\right\rangle_{\delta \phi}$,

$$
\left\langle n_{s}\right\rangle_{\phi}=\sum_{\left\{n_{j}\right\}} n_{s} P(\delta \phi)=\left\langle n_{s}\right\rangle \pm \frac{q \delta \phi}{k T}\left(\left\langle n_{s}\left(\sum_{s} z_{s} n_{s} \hat{v}_{s}^{b}\right)\right\rangle-\left\langle\sum_{s} z_{s} n_{s} \hat{v}_{s}^{b}\right\rangle\left\langle n_{s}\right\rangle\right),
$$

whereas at each site we must average over total particle number inside the site $\left\langle n_{s m}\right\rangle_{\phi}$

$$
\left\langle n_{s m}\right\rangle_{\delta \phi}=\sum_{\left\{n_{j}\right\}} n_{s m} P(\delta \phi)=\left\langle n_{s m}\right\rangle \pm \frac{q \delta \phi}{k T}\left(\left\langle n_{s m}\left(\sum_{s} z_{s} n_{s} \hat{v}_{s}^{b}\right)\right\rangle-\left\langle\sum_{s} z_{s} n_{s} \hat{v}_{s}^{b}\right\rangle\left\langle n_{s m}\right\rangle\right) .
$$

The resulting static density susceptibilities are written as,

$$
\begin{aligned}
& \chi_{s m}=\left(\left\langle n_{s m}\left(\sum_{s} \sum_{m} z_{s} n_{s m} v_{s m}^{b}\right)\right\rangle-\left\langle\sum_{s} \sum_{m} z_{s} n_{s m} v_{s m}^{b}\right\rangle\left\langle n_{s m}\right\rangle\right) \frac{1}{V_{s m} k T} . \\
& \chi_{s}=\left(\left\langle n_{s}\left(\sum_{s} \sum_{m} z_{s} n_{s m} v_{s m}^{b}\right)\right\rangle-\left\langle\sum_{s} \sum_{m} z_{s} n_{s m} v_{s m}^{b}\right\rangle\left\langle n_{s}\right\rangle\right) \frac{1}{V k T}
\end{aligned}
$$

We note that $V_{s m}$ is the site volume for each respective species $s$. The total volume of the pore is constant, but we allow the volume of each site to vary with species such that we can write $\sum_{m} V_{s m} \equiv V$. In both of these susceptibilities we have explicitly included correlations between ions of different species and between ions at different sites because both of these may be non-negligible. We can also write these functions using equilibrium statistical mechanics,

$$
\begin{aligned}
& \chi_{s m}=\left(\hat{v}_{s}^{b} z_{s} \frac{\partial\left\langle n_{s}\right\rangle}{\partial \Delta \bar{\mu}_{s m}}+\sum_{j \neq s}^{S} \hat{v}_{j}^{b} z_{j} \frac{\partial\left\langle n_{j}\right\rangle}{\partial \Delta \bar{\mu}_{s m}}\right) \frac{1}{V_{s m} k T} \\
& \chi_{s}=\left(\hat{v}_{s}^{b} z_{s} \sum_{m=1}^{M} \frac{\partial\left\langle n_{s}\right\rangle}{\partial \Delta \bar{\mu}_{s m}}+\sum_{j \neq s}^{S} \hat{v}_{j}^{b} z_{j} \sum_{m=1}^{M} \frac{\partial\left\langle n_{j}\right\rangle}{\partial \Delta \bar{\mu}_{s m}}\right) \frac{1}{V k T} .
\end{aligned}
$$

In total, the pore can be represented by a series of connected binding sites, and charge must be conserved meaning that current can only flow through the pore if all sites are conducting. Thus the pore is analogous to resistors in series and the resistivity $\mathcal{R}_{s m}$ and conductivity $\sigma_{s m}$ can be determined at each binding site. The reciprocal values of 
$\sigma_{s m}$ can be summed to obtain the total species conductivity. According to linear response theory the conductivity of a system can be calculated from the Einstein relation which relates the diffusivity $D$ to the susceptibility. This is derived in [10,11] for a system of volume $V$ as $\sigma=q^{2} D\left\langle(\Delta n)^{2}\right\rangle /(V k T)$ where the static density susceptibility is defined as $\chi=\left\langle(\Delta n)^{2}\right\rangle /(V k T)$. However, in our system $\chi_{s}$ or total variance in particle density may remain non-zero and even large when a binding site becomes non-conductive, which is in clear violation of Kirchoff's laws. Clearly, then, we need to determine the conductivity at each binding site in order to compute the total. Hence, we use the Einstein relation to calculate site-conductivity $\sigma_{s m}$ in terms of $\chi_{s m}$ Eqn. (31),

$$
\sigma_{s m}=z_{s} q^{2} \chi_{s m} D_{s m}
$$

Total species conductivity $\sigma_{s}^{T}$ can be computed, and this must be summed over species in order to obtain the total conductivity of the pore,

$$
\frac{1}{\sigma_{s}^{T}}=\sum_{m} \frac{1}{\sigma_{s m}}, \quad \sigma_{T}=\sum_{s} \sigma_{s}^{T}
$$

We note that if all ion types and sites become indistinguishable, then each site conductivity and hence $\sigma^{T}$ becomes proportional to the variance in total particle number as derived in $[10,11]$.

This result is in full agreement with numerous experimental examples within the literature. For example, point mutations affecting conduction in $\mathrm{K}^{+}$or $\mathrm{NaK}$ channels [12], or C-type inactivation in KcsA [13]. It is clear from Eqn. (31) that the conductivity is being written for a strongly correlated system, including single particle (self) and inter-particle correlations (perhaps between ions of different species). Furthermore, local pore volume can also determine conductivity/selectivity and the effects of mutation can be described.

Conductivity and hence electrical current can be defined at each site,

$$
\mathcal{G}_{s m}=\frac{A_{s m}}{L_{s m}} \sigma_{s m}, \quad I_{s m}=\mathcal{G}_{s m} \mathcal{V}_{m}
$$

where $A_{s m}, L_{s m}$ and $\mathcal{V}_{m}$ are surface area, length and applied voltage at each site $m$ respectively. Since total current across the whole pore equals current across each site, we can write the total current as,

$$
I_{s}=\mathcal{G}_{s}^{T} \mathcal{V}, \text { where: } \mathcal{G}_{s}^{T}=\left(\sum_{m}^{M} \frac{1}{\mathcal{G}_{s m}}\right)^{-1} .
$$

Here $\mathcal{V}=\sum_{m} \mathcal{V}_{m}$ is the total applied voltage.

If we consider a pore consisting of $M$ sites of identical geometry, and constant diffusivity throughout the pore, then the current simplifies to,

$$
I_{s}=\frac{z_{s} q^{2}}{L_{s m}^{2}} D_{s}^{c}\left(\sum_{m=1}^{M} \frac{1}{\tilde{\chi}_{s m}}\right)^{-1} \mathcal{V},
$$

where $L_{s m}=\left\langle L_{s m}\right\rangle$ is the average length of the binding site and $\tilde{\chi}_{s m}$ is the number susceptibility equal to $\chi_{s m}$ multiplied by the volume at each site (which is equal for each species and site in this instance). Furthermore, if each site shares binding affinity such that, $\tilde{\chi}_{s} \equiv \tilde{\chi}_{1}=\tilde{\chi}_{M}$, then one can write,

$$
I_{s}=z_{s} q^{2} \frac{D_{s}^{c}}{L_{s m}^{2}} \tilde{\chi}_{s} \mathcal{V}
$$

Let us consider our example of KcsA with 4 sites and a single conducting species. If site 4 has volume $V_{4}$ and sites 1-3 share volume $V_{m}$, then by introducing parameter $\beta$ defined as: $V_{4}=\beta V_{m}$, we can write,

$$
\sigma^{T}=\frac{D z q^{2}}{V_{m}} \frac{\tilde{\chi}_{1} \tilde{\chi}_{2} \tilde{\chi}_{3} \tilde{\chi}_{4}}{\beta \tilde{\chi}_{1} \tilde{\chi}_{2} \tilde{\chi}_{3}+\left(\tilde{\chi}_{1} \tilde{\chi}_{2} \tilde{\chi}_{4}+\tilde{\chi}_{1} \tilde{\chi}_{3} \tilde{\chi}_{4}+\tilde{\chi}_{2} \tilde{\chi}_{3} \tilde{\chi}_{4}\right)} .
$$

Interestingly when we consider the total conductance then this change in volume only affects the conductance if the length is varied. Thus, if we introduce $L_{4}=\gamma L_{m}$ we can write total conductivity as,

$$
\mathcal{G}^{T}=\frac{D z q^{2}}{L_{m}^{2}} \frac{\tilde{\chi}_{1} \tilde{\chi}_{2} \tilde{\chi}_{3} \tilde{\chi}_{4}}{\gamma \tilde{\chi}_{1} \tilde{\chi}_{2} \tilde{\chi}_{3}+\left(\tilde{\chi}_{1} \tilde{\chi}_{2} \tilde{\chi}_{4}+\tilde{\chi}_{1} \tilde{\chi}_{3} \tilde{\chi}_{4}+\tilde{\chi}_{2} \tilde{\chi}_{3} \tilde{\chi}_{4}\right)} .
$$


In the main paper we fit experimental current-voltage relations found for wild-type (WT) KcsA and a mutant (MuT). Mackinnon et. al. [14] states that the cysteine replaces threonine without significantly altering the side-chain volume. As a result we assume that all four sites S1-4 in the WT and S1-3 in the MuT have the same volumes, and consider S4 in the MuT to be slightly perturbed with $\gamma=1.2$. Furthermore we assume that both channels remain fully symmetrical with respect to the applied voltage, meaning that $\hat{v}_{m}^{b}=1 / 2$.

\section{ELECTROSTATICS AND EFFECTS OF IONIC BINDING}

In this section we shall briefly review the effects of ion binding, and the choice of electrostatics used within our theory.

\section{Effects of ion binding and polarisation}

The selectivity filter of KcsA is flexible, with structural fluctuations of $\sim 1 \AA$ r.m.s. [15], which are larger than the difference in the $\mathrm{Na}^{+} / \mathrm{K}^{+}$ion size. It is also known that the structure may change in response to the size and type of ion that is bound to the pore. For example at low $\mathrm{K}^{+}$concentrations the pore structure is altered because the Val-76 and Gly-77 residue adopt a new conformation resulting in the filter adopting a non-conducting "hour-glass" shape [16]. Smaller, more subtle effects in relation to polarisation are also expected to be important [17-20]. These effects can be considered explicitly within our theory, because the free energy contains both long and short range interactions which clearly depend on the pore structure. Furthermore, the average surface area and volume are used at various stages in the calculation of current. However, in its current application we have considered each site to share volume and structure regardless of the ionic configuration, and we will explore the effect of conformational changes in future applications.

\section{Electrostatic interaction}

In our energy expression Eqn. (12), the term $\mathcal{E}$ corresponds to the energy of electrostatic interaction between all the ions in state $\left\{n_{j}\right\}$ and all of the pore charges which total pore charge $n_{f}$. As stated in the main manuscript we are free to use any type of interaction, such as those used in Kitzing [21], or Yesylevskyy [22]; but we choose to consider the ICB-potential [23-26]. This potential takes account of the electric field being effectively reflected by the dielectric mismatch at the protein surface, analogously to light in an optical fiber. It is an assumption that is valid in the "short-channel" approximation. A "long" channel has a length exceeding twice the characteristic decay length, which is seven-times the radius of a water-filled pore [23]. Biological channels such as KcsA can be considered as short because their lengths are of order $10-15 \AA$, which is around the characteristic decay length. This interaction takes the form $Q^{2} / 2 C$ where $Q$ is total charge inside the pore and $C$ is its capacitance. We label it as interaction (1) for the purpose of comparison,

$$
\mathcal{E}^{(1)}=\frac{q^{2}\left(\sum_{s} z_{s} n_{s}+n_{f}\right)^{2}}{2 C} .
$$

We note that this interaction is identical to that used in the analogous study of electron transport through quantum dots, cf. Beenakker [27, 28].

As stated we are free to use any form of interaction, and we so have selected the most appropriate one. To demonstrate this let us consider a simple example: a 2 -site pore with up to 2 ions inside it. We compare the energy Eqn. (41) (with $n_{f}=-2.5$ ) to that of [22] which is comprised of two terms,

$$
\mathcal{E}^{(2)}=\sum_{n=1}^{N}\left[U\left(x_{n}\right)+\sum_{k>n}^{N} V\left(\left|r_{n}-r_{k}\right|\right)\right] .
$$

The first, $U$, is an inverted Gaussian potential designed to replicate the single-ion potential of mean force (PMF) of the pore,

$$
U(x)=-U_{0} e^{-(x / a)^{2}}
$$


We note that in this potential $U$ is designed to replicate the potential well created by attraction to the negatively charged pore, and parameters $U_{0}$ and $a$ corresponding to the value of the energy minima and width of the potential well, are determined by comparison to the single-ion potential of mean force (PMF). The second term, $V$, is the energy from the screened ion-ion Coulomb interaction between ions,

$$
V(r)=\frac{z_{1} z_{2} q^{2}}{4 \pi \epsilon_{0} r} e^{-r / d}
$$

Here: $x, d$ and $z_{s}$ correspond to the axial position in the pore, the shielding constant which is depenent on the electrical permittivity in the pore and the ionic valence. Although these are fitting parameters we shall simply consider the values used in [29]; and so $d=2.8 \AA, U_{0}=10.5 k T$ and $a=9 \AA$. Since Eqn. (42) assumes a pore centred on $x=0$ the ions occupy sites at $x=-2 \AA$ and $+2 \AA$ with the ion-to-ion separation being $4 \AA$ if both sites are occupied.

In Fig. 2 we compare the results using Eqns. (41) (top row) and (42) (bottom row), by plotting the total conductivity and occupancy vs. the affinities at each site $\Delta \tilde{\mu}_{m}$. Note that in the Conductivity Examples section, we discuss these surfaces in detail and provide a series of examples. Clearly the qualitative behaviour of the occupancy and conductivity is identical, with only a slight difference in the precise values of $\Delta \tilde{\mu}_{m}$ at which conduction occurs. This difference is to be expected because each potential contains a couple of fitting parameters, and so the energy barriers for the first and second ions to enter the pore slightly differ between the potentials. However, in each case the peaks form at the midpoint of the occupancy staircase when the difference in site affinities is small. We would expect similar qualitative agreement if we described the ion-pore interaction with $V$, and we shall analyse these interactions in a separate manuscript. One should not be surprised at the qualitative agreement between different potentials because the statistical properties are functions of the total energy and not simply of $\mathcal{E}$.
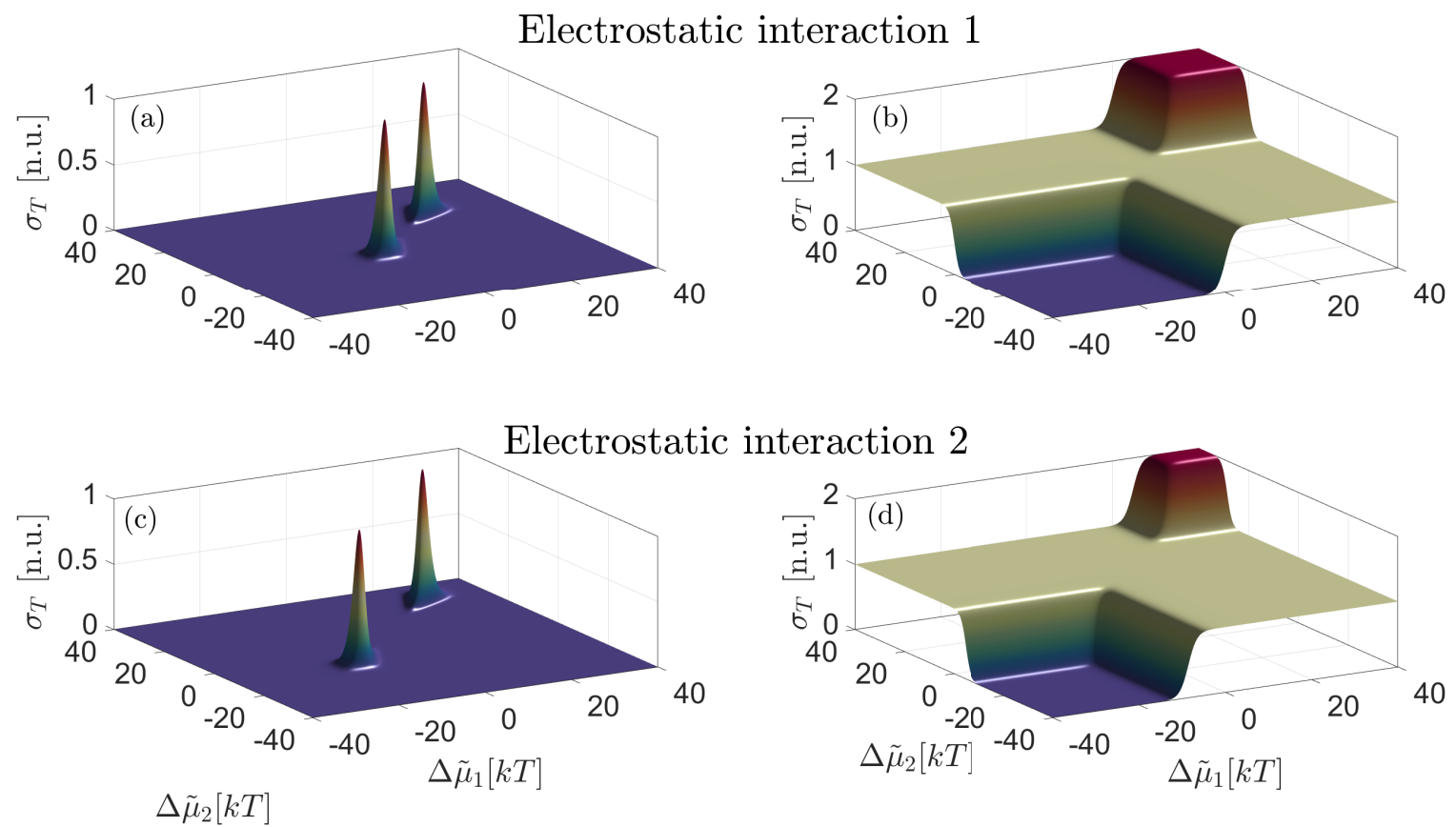

FIG. 2. Test of electrostatic interaction choice with potentials 1 and 2 given by Eqns. (41) and (42) respectively. (a) and (c) correspond to normalised total conductivity, meanwhile (b) and (d) are the total occupancies. With both potentials peaks form when the energy barrier to enter each site is close to being barrier-less and when the sites share affinity.

\section{NOVEL INSIGHTS FROM THE THEORY}

\section{Fundamental importance of structure}

The selectivity filter (SF) of a biological ion channel contains a large number of important parameters: number and position of binding sites, binding site geometry and volume, short-range interactions at each site and long-range 


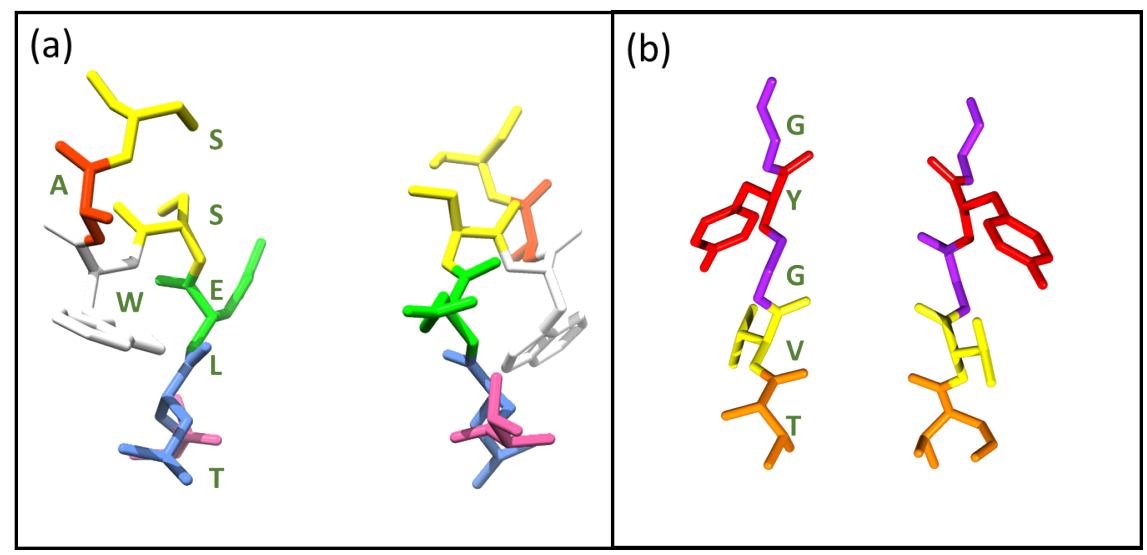

FIG. 3. Comparison of NaChBac [40] (a), and open KcsA (5vk6.pdb) (b) [2] visualised using chimera [3] and coloured by residue.

interactions in the pore [30-32]. Each of these is made more complicated by the facts that: the pore structure fluctuates; the electrical permittivity inside the pore is unknown; quantum mechanical interactions and polarisation of charge effects may occur; and these properties may vary with the ion type [17-20, 33, 34, 36-39, 81]. Furthermore, different channel types such as $\mathrm{K}^{+}, \mathrm{Na}^{+}, \mathrm{Ca}^{++}$channels or even slightly mutated channels have large differences in function. It is very clear that structure, and small changes in structure, can significantly affect the overall function of the pore.

In Fig. 3 we compare the structures of open KcsA (5vk6.pdb) [2] and the bacterial $\mathrm{Na}^{+}$channel $\mathrm{NaChBac}$ taken from [40]. Although the comparison is not to scale, it is known that $\mathrm{NaChBac}$ is roughly the same length as KcsA, but is wider, with a less uniform pore. Furthermore, $\mathrm{Na}^{+}$channels contain a strong and permanent glutatmate charge which directly interacts with the ions. However, its effective charge value is unknown due to possible polarisation and protonation effects [41-45]. The alteration in structure results in $\mathrm{NaChBac}$ favouring $\mathrm{Na}^{+}$over $\mathrm{K}^{+}$[44]. It is worth noting that the related $\mathrm{NaVaB}$ channel differs again in structure from $\mathrm{NaChBac}$, and is almost non-selective between $\mathrm{Na}^{+}$and $\mathrm{K}^{+}[46]$.

The importance of structure is perhaps most keenly observed in slightly mutated channels because, often, only a single residue is affected and yet it can lead to significantly altered channel properties. As described in the main paper, wild-type (WT) mutating (MuT) the threonine at residue 75 to cysteine does not significantly affect the sidechain volume but strongly reduces the occupancy of S4 and alters the electrostatic properties by removing 4 hydroxyl ligands [14]. In Fig. 4 we use a figure taken from [47] which compares the SF's of the WT in the closed configuration to the G77A and G77C mutants. Mutating the glycine at residue 77 had 3 interesting structural effects vs. the WT: the main-chain carbonyl group of V76 is prevented from pointing directly inwards into the channel's pore; the $\mathrm{K}^{+}$ ion at S2 is only coordinated by 4-main chain carbonyl groups from the A- or C-77 residues; and finally the SF's had a large reduction of occupancy at S1 in addition to S3 where the main mutation took place. The authors concluded that the ions must have been energetically coupled, thus stabilising the S2-S4 configuration. All three mutations also affect significantly the total conductivity of the pore. These three examples of mutated KcsA display vastly different function with both the site-occupancy and total conductivity being affected.

\section{Principal results of the main paper}

With these examples in mind we now consider Fig. 3 from the main text, which shows selective knock-on conduction within the pore. This figure is re-plotted in Fig. 5 with $\mathrm{K}^{+}$(blue) and $\mathrm{Na}^{+}$(red) occupancies (top) and conductivities (bottom) of (a), being plotted vs. $n_{f}$ and $\Delta \Delta \bar{\mu}_{K, N a ; S 4}$. We have also added 2-D cuts of total occupancy and species conductivity vs. $n_{f}$ in (b) and (c) respectively. In the cuts the solid lines are calculated with the extracted parameters for the WT and so $\Delta \bar{\mu}_{K, S 4}=6.2 k T$ and $\Delta \bar{\mu}_{N a, S 4}=0.2 k T$; and the blue and red stars correspond to the positions of $\mathrm{K}^{+}$and $\mathrm{Na}^{+}$conductivity predicted for KcsA. As discussed in the main text and in the examples below, the conductivity peak occurs at the degeneracy between neighbouring states, and thus we observe 3 steps and peaks corresponding to $0-1,1-2,2-3$ ion knock-on transitions. It is worth emphasising that the conductivity mechanism explicitly relies on the electrostatic correlations between ions and the structure of the pore. As can clearly be seen 

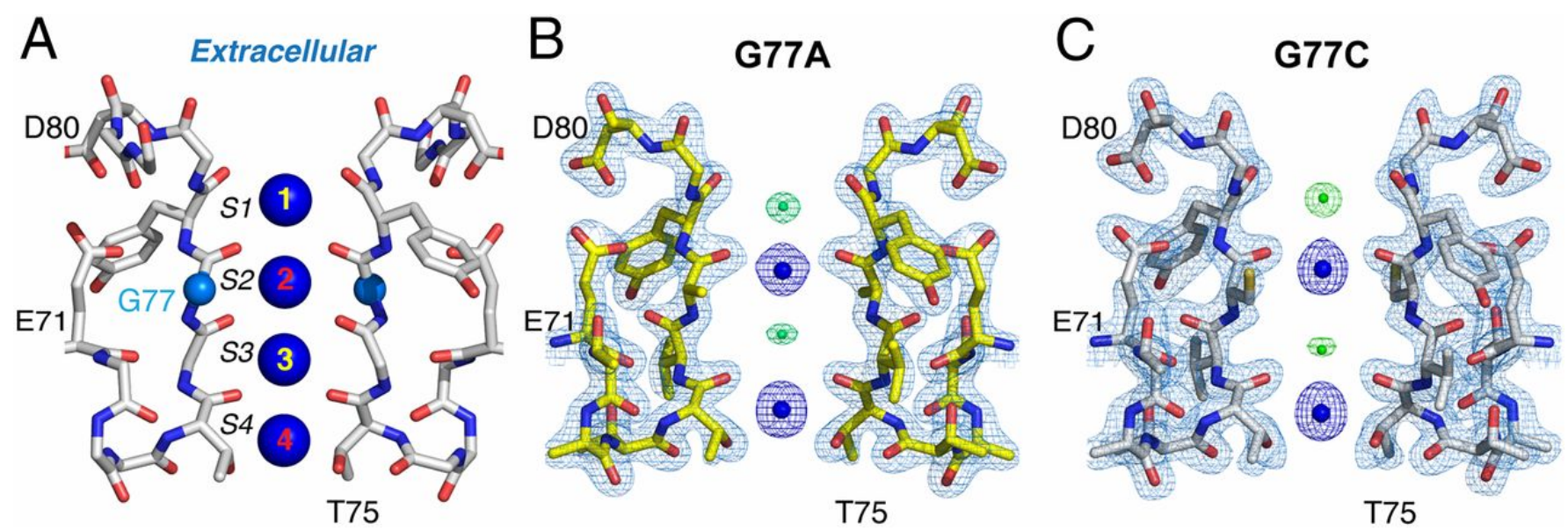

FIG. 4. Figure taken with permission from [47]. The closed wild-type KcsA channel (A) is compared to G77A (B) and G77C (C) mutations. In both mutations the main-chain carbonyl group of V76 is prevented from pointing directly inwards into the channel's pore, part of the coordinating oxygens at S2 have been removed, and the occupancy at S3 (and also S1) has been reduced alongside the overall conductivity.

from the figure, the total selectivity of the pore can be varied, and even reversed, simply by varying the selectivity of $\mathrm{S} 4$, although we note that the conductivity in this instance is negligible when compared to a $\mathrm{K}^{+}$favoured pore. For example, at $\Delta \Delta \bar{\mu}_{K, N a ; S 4}=-10 k T$ we find that the ratio of conductivity's $\sigma_{N a}^{T} / \sigma_{K}^{T}$ to be 17 in favour of $\mathrm{Na}^{+}$, however the overall conductivity is around $10^{4}$ times smaller than at the peak.

The predicted conductivity in the mutated channel is also plotted in the figures, and is shown by the green star. As a consequence of the mutation, we assumed the pore volume and affinity at S4 to change, resulting in $\Delta \bar{\mu}_{K, S 4}=5.2 \mathrm{kT}$. Therefore, the green star should not fit exactly onto the curve because this is calculated with parameters corresponding to the WT: it is simply a guide to the reader. In fact, this highlights the two contributors to the reduced conductivity: (1) a reduced pore charge and (2) a difference in site affinity/geometry. The green star is below the theoretical line and so the difference in site affinity/geometry reduces the conductivity of the mutant relative to the WT at $n_{f}=-2.32$ by around $25 \%$. However, the overall reduction in conductivity of the MuT vs. the WT is increased by the change in $n_{f}$, with the overall MuT/WT conductivity ratio being $\sim 0.075$.

\section{Proposed future work}

A subset of readers may find it useful to know how our general theory can be extended in the future, and we outline some promising directions below -

- Different binding sites for different species. $\mathrm{Na}^{+}$ions have been observed to bind at different sites within the pore $[20,33,34,81]$. These sites, form in the plane of the coordinating ligands and are $\mathrm{Na}^{+}$selective. The effects of additional binding sites, and their potential role in selectivity [12], can be investigated and explored within our theory, simply by increasing the number of available binding sites and hence states of the system, and considering a different selectivity. For example, adding 1 planar site would increase the number of states for $0-3 \mathrm{~K}^{+}$or $\mathrm{Na}^{+}$ions in the pore from 65 to 111. It is important to note that the derivation of occupancy and conductivity remains the same, and so one would simply need to recalculate these properties.

- The optimal conduction mechanism in KcsA. Currently, there is debate as to the nature of the conduction mechanism inside KcsA [4, 48-50]. In our main paper, we only consider 0-3 ions inside the pore and observe 2-3 ion conduction with $n_{f}=-2.5$, based on the ideas of MacKinnon [51]. We can equally easily consider 0-4 ions in the pore, however, and identify the range of pore properties required to observe either $2-3$ or $3-4$ ion conduction events.

- Modelling the $\mathbf{K}^{+}$channel family. It is known that $\mathrm{K}^{+}$channels, which are likely to be present in every living being [52], have conserved selectivity filters sharing the xxGYG sequence and, in most cases, sharing the full TVGYG sequence[82]. Despite, the similarities in structure of the SF, these channels can have vastly different conductivity and selectivity properties, for example the conductivity can vary from 5 to $270 \mathrm{pS}$ [54]. It 
has been shown experimentally that the cavity contributes significantly to the pore resistance, thus lowering the conductance of Shaker; and this conductance is increased when mutations are made to the cavity [55]. Thus, the cavity is expected to play a key role in determining the differences of conductance $[54,55]$. Wee plan to incorporate the cavity into our theory by adding it as a separate subsystem. We would be free to alter the interaction strength and cavity geometry, and could test if varying the cavity dimensions significantly affects the overall conductivity, or if changes to the binding affinities in the cavities are also needed.

- Developing a Kinetic model and deriving self-consistent transition rates. Kinetic models are widely used to investigate the ionic currents and selectivities far from equilibrium [56-58]. However, such theories all depend on ionic transition rates which, unlike their electronic counterparts, can not easily be rigorously derived and, as a result, are a source of criticism [59,60]. Our theory provides an excellent basis on which to derive these rates, as we have shown recently [61]. The kinetic model starts from the set of states used in the statistical theory and, by comparing the current at linear response to the current in our manuscript, one can identify the form of transition rates required. In [61] we developed this logic through application to NaChBac. Note, however, that we considered a simplified geometry and neglected the effects of correlations between ions at different sites. Further work is needed.

- Incorporating polarisation. At present most of the available force fields, such as AMBER or CHARM [6265], do not include polarisation. Yet ions inside the pore may face localised electrostatic effects so that account should be taken of $[66,67]$. In our theory each binding state is characterised by the location, number and type of ions present inside a pore with a given structure, as well as the local electrical permittivity. Hence there are several possible approaches to taking account of polarisation. The simplest one is to vary the local polarisation in our energy term due to the binding state of the pore. Alternatively we can modify the parameters separately for ion-ion and ion-fixed charge interactions. At a more fundamental level, the state of polarisation is included within multi-ion PMFs calculated using polarisable force-fields. Accordingly, the energy term can be calculated explicitly using polarised multi-ion PMFs. We plan to estimate analytically a multi-ion PMF that includes polarisation. We will do so by extending the results from [68, 69], and using the Drude model introduced by Roux [70, 71]. In addition polarisation can be included explicitly within the linear response formalism [11] via fluctuations of energy which account for the changes in structure and polarisation.

Even in its current form, as presented in the main paper, the theory illuminates the transport properties of narrow biological channels quite generally, including in particular the $\mathrm{K}^{+}$-channel family - and it also provides a robust basis for future developments.

\section{CONDUCTIVITY EXAMPLES}

The occupancy and conductivity highlighted by Fig. 3 of the main paper, exhibit rich and complex behaviour. To explore and explain these phenomena in the greater detail that a subset of readers may find useful, we now consider 3 important examples outlining the conductivity-occupancy relationship, and the effects of allowing more ions and ion types inside the pore. Note that we assume KcsA geometry with 4 sites and a total channel length and radius of $12 \AA$ and $2 \AA$ respectively. Although not necessary, we also consider the limit where all site volumes are identical and equal to the total pore volume divided by the number of sites, $V_{s m} \equiv V^{c} / M$, and where the diffusivities are also equal in each site.

\section{Example 1: One species, one ion, multiple binding sites}

In this example we investigate the system under the 0-1 ion transport mechanism, for a pore with two binding sites. We consider the binding affinity in site 1 to be fixed but mutate site 2 and thus allow $\Delta \tilde{\mu}_{2}=\Delta \bar{\mu}_{2}+k T \log (x)$ to vary in accordance with Fig. 3 of the main paper. The aim is to describe the effect on the conduction mechanism of the interplay of distinguishable binding sites. In this system the partition function comprises of one empty and two singly-occupied states. We denote the number of ions and their location within the free energy,

$$
Z=e^{-G(0) / k T}+e^{-G(1 K, m=1) / k T}+e^{-G(1 K, m=2) / k T}
$$

In Fig. 6 we consider the energy spectra $v s$. charge of the pore $n_{f}$. The black dashed curve represents the state without ions; the purple and green curves represent states with an ion at site 1 and 2 respectively. When the ion is in 
site 2 we showcase the spectra when $\Delta \tilde{\mu}_{2}=1.5 k T$. Coloured circles highlight the occurrence of degeneracy between the zero and strongly occupied states. At each of these points we find that the respective energy barrier is zero i.e. $\Delta G=0$. Here, the system is degenerate because either state is equally probable and, under certain conditions that we will elucidate later, may yield conduction. Due to the difference in $\Delta \tilde{\mu}$, splitting between the single ion states occurs, resulting in a shift of the degeneracy point. However, it is clear that when such a degeneracy occurs at a higher energy than another i.e. the purple curve in this figure; then the probabilities of the states in question are smaller than the probability for the ion to be in site 2. Thus we can establish the following energy barrier at each degeneracy point,

$$
\Delta G_{m}=\Delta \mathcal{E}+k T \log \left[(n+1) / n_{0}\right]-\Delta \bar{\mu}_{m}-k T \log (x)=0 .
$$

Let us consider the occupancy and conductivity of each site (see Fig. 7). Occupancy ((a) and (c)) forms a step-like function vs. both $\Delta \tilde{\mu}_{2}$ and $n_{f}$ labelled in the figure as step 1 and 2 respectively. Step 1 also corresponds to a step in total particle number, but step 2 does not because here the single ion states are always favoured and it is just the pore favouring site 1 or 2 . The conductivity forms a sharp peak close to the midpoint of step 1 . Therefore, we can conclude that conduction maximises at the degeneracy condition (46) and when the site is favoured. When a difference between site affinities exists, conduction may still occur but it is reduced.

The total conductivity and occupancy are considered in Fig. 8. Panels (a) and (b) show the total occupancy and normalised conductivity through the pore vs.n $n_{f}$ and $\Delta \tilde{\mu}_{2}$. It is clear from both figures that the $\Delta \bar{\mu}_{2}=0$ plane is important because it is around this that conductivity is peaked and where occupancy starts to vary due to the growing importance of the state with the ion occupying site 2 .

Total conductivity is given by the inverse of the sum of the reciprocal conductivities at individual sites. For this example we can write,

$$
\sigma^{T}=\frac{\sigma_{1} \sigma_{2}}{\sigma_{1}+\sigma_{2}}
$$

where we remind the reader that each conductivity is proportional to the susceptibility into the given site. Therefore as the difference in affinity between sites grows i.e. the limit $\left|\Delta \tilde{\mu}_{1}-\Delta \tilde{\mu}_{2}\right| \gg 0$ the susceptibility and conductivity into the least favoured site tends to zero and the total conductivity drops to zero. Thus, in contrast to conduction into the individual sites, conduction now occurs close to the degeneracy condition (46) provided that the difference in site affinities is small $\left|\Delta \tilde{\mu}_{1}-\Delta \tilde{\mu}_{2}\right| \sim 0$. In fact, it is not exactly zero because we have omitted a small factor $\epsilon$ due to the entropy of mixing of binding sites.

To gain further insight, we now consider the conductivity and occupancy of site 1 only and examine the limit that site 1 is more favoured than site 2 i.e. $\Delta \tilde{\mu}_{1} \gg \Delta \tilde{\mu}_{2}$,

$$
\begin{gathered}
\left\langle n_{1}\right\rangle=\frac{e^{-\Delta G_{1} / k T}}{1+e^{-\Delta G_{1} / k T}} \\
\sigma_{1} \propto \frac{e^{-\Delta G_{1} / k T}}{\left(1+e^{-\Delta G_{1} / k T}\right)^{2}} .
\end{gathered}
$$

Here, $\Delta G_{m}=G(1 K, m)-G(0)$. Therefore it is evident that conductivity into each site maximises at the midpoint of the occupancy step, i.e. $\Delta G_{m}=0$ when this is the favoured site. If we consider the total occupancy and conductivity then we would find,

$$
\begin{aligned}
& \langle n\rangle \rightarrow\left\langle n_{1}\right\rangle \\
& \sigma \rightarrow 0 .
\end{aligned}
$$

If we consider the total conductivity and occupancy when the site affinities are equal i.e. $\Delta G_{1}=\Delta G_{2}=\Delta G$ then we can write,

$$
\begin{gathered}
\langle n\rangle=\frac{2 e^{-\Delta G / k T}}{1+2 e^{-\Delta G / k T}} \\
\sigma \propto \frac{e^{-\Delta G / k T}}{\left(1+2 e^{-\Delta G / k T}\right)^{2}} .
\end{gathered}
$$

Therefore, it is clear the the peak of the total conductivity is slightly offset from the condition $\Delta G=0$, due to a factor of $k T \ln (2)$. This occurs due to the degeneracy between sites which results in multiple (2 in this instance) 
possible transitions. However, these contributions are often small and so we neglect them and write the following two conditions for maximal conductivity,

$$
\begin{aligned}
& \Delta G=0 \\
& \Delta \bar{\mu}_{s 1} \sim \Delta \bar{\mu}_{s m^{\prime}}, \in m^{\prime}=2, \cdots, M
\end{aligned}
$$

Finally it is worth noting that here the fixed charge here plays an identical role to that of the gate voltage in quantum dots and transistors. As a result the conductivity peak and occupancy step correspond to ionic Coulomb blockade. This ionic analogue of electronic Coulomb blockade has been already been identified as an important classical ionic transport process found in artificial and biological channels [25, 26, 72, 73]. This is clearly observed in (c) of Fig. 8 where two traces at $\Delta \tilde{\mu}_{2}=0$ and $\Delta \tilde{\mu}_{2}=1.5 k T$ produced shifted conductivity peaks and Coulomb steps in occupancy.

\section{Example 2: One species, two ions, two binding sites}

In this next example we shall restrict ourselves to a single species, but allow up to two ions inside the pore. The aim is to investigate changes in conductivity when varying the number of ions inside the pore and the conduction mechanism i.e. 0-1 to 1-2 transitions through the pore. The parameters are identical to those used in the previous example. The partition function is slightly extended to include a fourth double ion state,

$$
Z=e^{-G(0) / k T}+e^{-G(1 K, m=1) / k T}+e^{-G(1 K, m=2) / k T}+e^{-G(2 K, m=1,2) / k T}
$$

In Fig. 9 we plot the energy spectra again vs. $n_{f}$. Purple and green curves correspond respectively to single ions at sites 1 and 2 when $\Delta \tilde{\mu}_{2}=+1.5 k T$. The black dashed line is again the state with zero ions and the red spectrum corresponds to ions in both sites and again $\Delta \tilde{\mu}_{2}=+1.5 k T$. We covered the first transition in the earlier example and so here we shall focus on the second.

We consider the occupancy and fluctuations in (a) and (b) of Fig. 10 respectively. Both functions have an antisymmetrical dependence on the $\Delta \tilde{\mu}_{2}=0$. This is because after the first transition, and unless $\Delta \tilde{\mu}_{1} \sim \Delta \tilde{\mu}_{2}$, one site is more likely to be occupied than the other. To observe this effect we have plotted the occupancy and conductivity of site 2 when the sites share affinity (c) and when site 1 is more favoured by $1.5 \mathrm{kT}(\mathrm{d})$. When sites share binding affinity the movement of ions into site 2 is equally probable for both the $0-1$ and 1-2 transitions. As a result the conductivity peaks and occupancy steps are equal in magnitude. However, when site 2 is favoured in (d) we can clearly see the effects of the energy barrier prohibiting the ion from entering site 2 if the pore already contains an ion because its conductivity is reduced.

We can now also investigate the periodicity of the peaks along the $n_{f}$ plane. Since $\Delta \tilde{\mu}$ is unchanged with differing states of the system the periodicity is largely defined by the difference in the longer-range electrostatic interactions of the pore $\Delta \mathcal{E}$. Thus with the approximation used corresponds to the renormalised energy difference in the absence of charging effects: $2 U_{c}[27]$. This is easily seen in Fig 9 if you consider the energy difference between the red and green curves during the first transition.

\section{Example 3: Two species, multiple ions, multiple binding sites}

In our third example we consider the effects of competition inside the pore by allowing for two conducting species, sodium $\mathrm{Na}^{+}$and potassium $\mathrm{K}^{+}$. We take: $U_{c}=10 k T, x_{K}=x_{N a}, \Delta \tilde{\mu}_{1 K / N a}=0$ for both $\mathrm{K}^{+}$and $\mathrm{Na}^{+}$and allow $\Delta \Delta \tilde{\mu}_{K, N a ; S 2}$ to vary (thus mimicking the conditions used in Fig. 3 of the main paper). In this system the partition function is comprised of the following states,

$$
\begin{gathered}
Z=e^{-G(0) / k T}+e^{-G(1 K, m=1) / k T}+e^{-G(1 K, m=2) / k T}+e^{-G(1 N a, m=1) / k T}+e^{-G(1 N a, m=2) / k T}+ \\
e^{-G(2 K, m=1,2) / k T}+e^{-G(2 N a, m=1,2) / k T}+e^{-G(1 K, m=1 ; 1 N a, m=2) / k T}+e^{-G(1 K, m=2 ; 1 N a, m=1) / k T}
\end{gathered}
$$

We start by considering the energy spectra in Fig. 11. The solid purple and green curves correspond to pure $\mathrm{K}^{+}$and $\mathrm{Na}^{+}$states, the dashed orange represents either $\mathrm{K}^{+}$or $\mathrm{Na}^{+}$at site 1 , the dashed yellow line is either $\mathrm{K}^{+}$or $\mathrm{Na}^{+}$at site 1 and $\mathrm{K}^{+}$at site 2 , and finally the black line denotes the zero-ion state. There is a major difference to the earlier examples because the energy spectra can now undergo splitting due to the ion type in addition to the configuration i.e. sites occupied. As outlined in the central result of the main paper, selectivity at each site can be determined for a fixed $n_{f}$ and in the case of symmetrical solutions it yields the Eisenman relation $\left|\Delta \tilde{\mu}_{s m}-\Delta \tilde{\mu}_{j m}\right|$. We also classify 
higher order $(n>1)$ states into either pure i.e. $2 \mathrm{~K}^{+}$or mixed $1 \mathrm{~K}^{+} 1 \mathrm{Na}^{+}$states. This is apparent from the dashed purple curve which is the lowest-energy mixed state (the other is not shown) corresponding to $\mathrm{K}^{+}$at site 2 and $\mathrm{Na}^{+}$ at site 1 . As a result mixed species conduction can now occur under specific parameters via pure or mixed states.

In Fig. 12 we consider the total occupancy (a) and conductivity (b) through the pore. As before, an ion can enter any free site. Thus conduction is again governed by which site has the higher affinity. However, this is not the complete picture because sites can be selective and by differing amounts. Thus in the limit that an ion can only enter site $m$, and this site is strongly favoured for species $i$, one would expect conduction to occur at the degeneracy condition for this site and species (again neglecting the small mixing term). So in our example $\mathrm{K}^{+}$maximally conducts when $\Delta \tilde{\mu}_{2} \sim 1.5 k T$. If the sites are not strongly selective or if selectivity differs at some sites meaning that multiple possible conduction events are possible then conduction is not maximal unless the system is completely isoenergetic and non-selective.

So we can conclude that conduction is governed by strict conditions. If we are interested in maximal and selective conduction for ions of species $i$ then we must require that the following conditions are satisfied,

$$
\begin{aligned}
& \Delta G_{m}=0 . \\
& \Delta \bar{\mu}_{s 1} \sim \Delta \bar{\mu}_{s m^{\prime}}, \in m^{\prime}=2, \cdots, M \\
& \Delta \bar{\mu}_{s m} \gg \Delta \bar{\mu}_{j m}, \in m=2, \cdots, M .
\end{aligned}
$$

\section{RELATION TO PMF}

To derive a relationship between our discrete space free energy $G$ and the continuous multi-ion PMFs calculated from simulation we shall consider the statistical theory developed by Roux [74, 75]. In this work the binding factor $\mathcal{B}_{n}$ was introduced, representing the ratio of probabilities to have $n$ and 0 ions within the pore. We shall limit ourselves to a single species occupying up to $M$ binding sites in the pore. In Roux's theory the binding factor was explicitly formulated in terms of the multi-ion PMF $\mathcal{W}$,

$$
\mathcal{B}_{n}=\frac{\left(\bar{\rho}^{I}\right)^{n}}{n !} \times \int_{c} d \mathbf{r}_{1} \cdots \int_{c} d \mathbf{r}_{n} e^{\left[n \bar{\mu}^{(I)}-\mathcal{W}\left(r_{1}, \cdots r_{n}\right)\right] / k T},
$$

such that $\mathcal{B}_{0}=1$. In his notation $n$ is the number of ions in the pore, $\bar{\rho}^{I}$ is the density of permeable ions in bulk $I$ and $\bar{\mu}^{(I)}$ is the excess chemical and electrical potential. As a result, we find in our notation that $\mu^{b} \sim k T \log \left(\bar{\rho}^{I}\right)+\bar{\mu}^{(I)}$. The PMF is continuous tracking the positions of all the ions across the whole pore. This differs from our theory which consider the same number of ions in a set of different configurations as ions occupy any binding sites. As a result we can introduce the probability to find $n$ ions inside the pore by summing over all possible configurations that contain $n$ ions,

$$
P_{n}=\sum_{\left\{n_{j}\right\}=n} P\left(\left\{n_{j}\right\}\right)
$$

and the same is true for the free energy,

$$
G_{n}=\sum_{\left\{n_{j}\right\}=n} G\left(\left\{n_{j}\right\}\right) .
$$

As a result in our theory the binding factor takes the following form,

$$
\mathcal{B}_{n}=e^{\left(G_{0}-G_{n}\right) / k T} .
$$

Through comparison of binding factors we are able to recover the following relationship,

$$
\sum_{\left\{n_{j}\right\}=n} e^{\left[G_{0}-G\left(\left\{n_{j}\right\}\right)\right] / k T}=\frac{1}{n !} \int_{c} d \mathbf{r}_{1} \cdots \int_{c} d \mathbf{r}_{n} e^{\left[n \mu^{b}-\mathcal{W}\left(r_{1}, \cdots r_{n}\right)\right] / k T} .
$$

If we cancel the $n$ ! terms we are left with,

$$
+k T \log \left(\frac{1}{n_{0} !}\right)+k T \log \left[\sum_{\left\{n_{j}\right\}=n} e^{\left[\left(\mathcal{E}(0)-\mathcal{E}\left(\left\{n_{j}\right\}\right)+\sum_{m} n_{m} \Delta \tilde{\mu}_{m}\right] / k T\right.}\right]=+k T \log \left(\int_{c} d \mathbf{r}_{1} \cdots \int_{c} d \mathbf{r}_{n} e^{\left[n \mu^{b}-\mathcal{W}\left(r_{1}, \cdots r_{n}\right)\right] / k T}\right) .
$$


where $n_{0}$ is the number of empty sites with $n$ ions in the pore. In this relationship $\mathcal{E}\left(\left\{n_{j}\right\}\right)$ represents the total electrostatic energy produced from all the interactions between ions and pore charges when the ions are in the specified configuration $\left\{n_{j}\right\}$. Finally we note that in the limit that there is only one configuration i.e. if $n=1$ and $M=1$, then we find,

$$
-\Delta \mathcal{E}+\Delta \tilde{\mu}=+k T \log \left(\int_{c} d \mathbf{r}_{1} e^{\left[\mu^{b}-\mathcal{W}\left(r_{1}\right)\right] / k T}\right) \text { or }-\Delta \mathcal{E}-\bar{\mu}^{c}=+k T \log \left(\int_{c} d \mathbf{r}_{1} e^{-\mathcal{W}\left(r_{1}\right) / k T}\right) .
$$

In the second equation we have cancelled $\mu^{b}$ from both sides.

\section{ANALYSIS OF PARAMETERS}

The two key parameters within our theory are $\Delta \bar{\mu}_{K m}$ and $\Delta \bar{\mu}_{N a m}$, each of which represents the difference in excess chemical potential between the bulk reservoir and site $m$. We derive $\Delta \bar{\mu}_{K m}$ for sites S1-S4 through fitting of experimentally calculated occupancy's and current-voltage relations. In the main text we have established these parameters for both the wild type (WT) and the mutant (MuT). If we approximate $\bar{\mu}_{K}^{b}$ to be equal to the hydration energy in the bulk $-120 k T$ for $\mathrm{K}^{+}[76]$ we can estimate the value of the potential within the channel and these values are given in table I. Krishnapriya et. al. performed free energy calculations the $1 \mathrm{~K} 4 \mathrm{C}$ (closed) structure using the AMBER force field to calculate the binding energies at each site (see the paper for details). The authors also found that accounting for quantum mechanical effects only slightly varied the binding energies. The binding energies at S1-S3 were found to be $-68,-85$ and $-117 k T$ respectively and it was found that $\mathrm{K}^{+}$cannot bind to S4. The latter result has also been observed in [77] who found that S4 cannot be occupied if the two ions are at S1 and S3. It is worth noting that these values strongly vary in contrast to the experimental occupancies which slightly fluctuate around $\sim 0.5$ and occupancy of S4 has been observed [14]. Asthagri et. al. [78] calculated the excess chemical potential in S2 using the potential distribution theorem with two potassium ions placed inside the pore. The average excess chemical potential was found to be $\sim-150 k T$ for $\mathrm{K}^{+}$and $\sim-180 k T$ for $\mathrm{Na}^{+}$although we note that the bulk hydration energies for $\mathrm{K}^{+}$and $\mathrm{Na}^{+}$are different and not included here.

Site selectivity is determined within our theory by the difference in excess chemical potential difference, and hence includes the difference in site affinity in addition to the difference in solvation energy within the bulk reservoirs. In $[15,79-81]$ the site selectivities were calculated using molecular dynamics techniques on the closed 1K4C.pdb KcsA channel. Typically three K ions were taken to be in either S1-S4 or possibly in site S0 (a fifth extra-cellular sided site observed in simulation) or in the cavity site close to the pore and a single ion is replace by $\mathrm{Na}^{+}$. The corresponding free energy change is quoted as the selectivity. Although [81] also considered the selectivities at various locations within the pore we only consider the values in the oxygen cages that are known to be $\mathrm{K}^{+}$binding sites. These calculations were performed at differing temperatures and so we convert them into values of $\mathrm{kT}$ at $300 \mathrm{~K}$. The values in the table vary significantly both in terms of magnitude but also sign with $\mathrm{S} 4$ in $[15,79]$ being shown to be $\mathrm{Na}^{+}$selective. As a result we consider the values from [81] to calculate $\Delta \bar{\mu}_{N a, m}$.

\begin{tabular}{|c|c|}
\hline Site & Our theory $\bar{\mu}_{m K}^{c, W T}[k T]$ \\
\hline$m=1(\mathrm{~S} 1)$ & -126.2 \\
\hline$m=2(\mathrm{~S} 2)$ & -125.7 \\
\hline$m=3(\mathrm{~S} 3)$ & -126 \\
\hline$m=4(\mathrm{~S} 4)$ & -126.2 \\
\hline
\end{tabular}

TABLE I. Local interaction at the site in units of $k T$.

\begin{tabular}{|c|c|c|c|}
\hline Site & From $[15,79][k T]$ & From $[81][k T]$ & From $[78]$ and $([80])[k T]$ \\
\hline$m=1(\mathrm{~S} 1)$ & +4.2 & +4 & $(+7.7)$ \\
\hline$m=2(\mathrm{~S} 2)$ & +8.5 & +8.3 & +4.5 \\
\hline$m=3(\mathrm{~S} 3)$ & +2.9 & +7.6 & N/A \\
\hline$m=4(\mathrm{~S} 4)$ & -2 & +6.1 & N/A \\
\hline
\end{tabular}

TABLE II. Selectivities at each site in units $k T$. 
*w.gibby@lancaster.ac.uk

† dmitry.g.luchinsky@nasa.gov

‡ p.v.e.mcclintock@lancaster.ac.uk

[1] D. M. Rogers, T. L. Beck, and S. B. Rempe, "An information theory approach to nonlinear, nonequilibrium thermodynamics," J. Stat. Phys., vol. 145, no. 2, pp. 385-409, 2011.

[2] L. G. Cuello, D. M. Cortes, and E. Perozo, "The gating cycle of a k+ channel at atomic resolution," Elife, vol. 6, p. e28032, 2017.

[3] E. F. Pettersen, T. D. Goddard, C. C. Huang, G. S. Couch, D. M. Greenblatt, E. C. Meng, and T. E. Ferrin, "Ucsf chimera - a visualization system for exploratory research and analysis," J. Comput. Chem., vol. 25, no. 13, pp. 1605-1612, 2004 .

[4] D. Doyle, J. Cabral, R. Pfuetzner, A. Kuo, J. Gulbis, S. Cohen, B. Chait, and R. MacKinnon, "The structure of the potassium channel: Molecular basis of $\mathrm{K}^{+}$conduction and selectivity," Science, vol. 280, no. 5360, pp. 69-77, 1998.

[5] B. Widom, "Potential-distribution theory and the statistical mechanics of fluids," J. Phys. Chem., vol. 86, no. 6, pp. 869$872,1982$.

[6] B. Roux, T. Allen, S. Berneche, and W. Im, "Theoretical and computational models of biological ion channels," Quart. Rev. Biophys., vol. 37, no. 1, pp. 15-103, 2004.

[7] D. Gillespie, "Energetics of divalent selectivity in a calcium channel: The ryanodine receptor case study," Biophys. J., vol. 94, no. 4, pp. 1169-1184, 2008.

[8] J. G. Kirkwood, "Statistical mechanics of fluid mixtures," J. Chem. Phys., vol. 3, no. 1935, pp. 300-313, 1935.

[9] R. Zwanzig, Nonequilibrium statistical mechanics. Oxford University Press, 2001.

[10] R. Kubo, "Statistical-mechanical theory of irreversible processes. i. general theory and simple applications to magnetic and conduction problems," J. Phys. Soc. Jpn., vol. 12, no. 6, pp. 570-586, 1957.

[11] R. Kubo, "The fluctuation-dissipation theorem," Reps, Progr. Phys., vol. 29, no. 1, p. 255, 1966.

[12] M. Derebe, D. Sauer, W. Zeng, A. Alam, N. Shi, and Y. Jiang, "Tuning the ion selectivity of tetrameric cation channels by changing the number of ion binding sites.," Proc. Nat. Acad. Sci. (USA), vol. 108, no. 2, pp. 598-602, 2011.

[13] Y. Xu and A. E. McDermott, "Inactivation in the potassium channel KcsA," J. Struct. Biol. X, vol. 3, p. 100009, jul 2019.

[14] M. Zhou and R. MacKinnon, "A mutant KcsA $\mathrm{K}^{+}$channel with altered conduction properties and selectivity filter ion distribution," J. Mol. Biol., vol. 338, no. 4, pp. 839-846, 2004.

[15] S. Noskov, S. Berneche, and B. Roux, "Control of ion selectivity in potassium channels by electrostatic and dynamic properties of carbonyl ligands," Nature, vol. 431, no. 7010, p. 830, 2004.

[16] Y. Zhou, J. Morais-Cabral, A. Kaufman, and R. MacKinnon, "Chemistry of ion coordination and hydration revealed by a $\mathrm{K}^{+}$channel-Fab complex at $2.0 \AA$ resolution," Nature, vol. 414, no. 6859, pp. 43-48, 2001.

[17] S. Varma and S. B. Rempe, "Multibody effects in ion binding and selectivity," Biophys. J., vol. 99, no. 10, pp. 3394-3401, 2010.

[18] S. Varma, D. M. Rogers, L. R. Pratt, and S. B. Rempe, "Design principles for $\mathrm{K}^{+}$selectivity in membrane transport," J. Gen. Physiol., vol. 137, no. 6, pp. 479-488, 2011.

[19] D. Bucher and U. Rothlisberger, "Molecular simulations of ion channels: a quantum chemist's perspective," J. Gen. Physiol., vol. 135, no. 6, pp. 549-554, 2010.

[20] S. De, C. Rinsha, A. Joseph, A. Ben, V. Krishnapriya, et al., "Roles of different amino-acid residues towards binding and selective transport of k+ through kcsa k+-ion channel," Phys. Chem. Chem. Phys., vol. 20, no. 25, pp. 17517-17529, 2018.

[21] E. Kitzing, "A novel model for saturation of ion conductivity in transmembrane channels," in Membrane Proteins: Structures, Interactions and Models: Proc. 25th Jerusalem Symposium on Quantum Chemistry and Biochemistry, Jerusalem, May 18-21,1992 (A. Pullman, J. Jortner, and B. Pullman, eds.), pp. 297-314, Dordrecht: Springer Netherlands, 1992.

[22] S. Yesylevskyy and V. Kharkyanen, "Barrier-less knock-on conduction in ion channels: peculiarity or general mechanism?," Chem. Phys., vol. 312, pp. 127-133, 2005.

[23] J. Zhang, A. Kamenev, and B. I. Shklovskii, "Conductance of ion channels and nanopores with charged walls: A toy model," Phys. Rev. Lett., vol. 95, p. 148101, 2005.

[24] J. Zhang, A. Kamenev, and B. I. Shklovskii, "Ion exchange phase transitions in water-filled channels with charged walls," Phys. Rev. E, vol. 73, p. 051205, 2006.

[25] M. Krems and M. Di Ventra, "Ionic Coulomb blockade in nanopores," J. Phys. Condens. Matter, vol. 25 , p. 065101, 2013.

[26] I. K. Kaufman, P. V. E. McClintock, and R. S. Eisenberg, "Coulomb blockade model of permeation and selectivity in biological ion channels," New J. Phys., vol. 17, no. 8, p. 083021, 2015.

[27] C. Beenakker, "Theory of Coulomb-blockade oscillations in the conductance of a quantum dot," Phys. Rev. B, vol. 44, no. 4, pp. 1646-1656, 1991.

[28] E. Bonet, M. M. Deshmukh, and D. Ralph, "Solving rate equations for electron tunneling via discrete quantum states," Physical Review B, vol. 65, no. 4, p. 045317, 2002.

[29] M. Barabash, W. A. T. Gibby, C. Guardiani, D. Luchinsky, and P. V. E. McClintock, "From the potential of the mean force to a quasiparticle's effective potential in narrow ion channels," Fluct. Noise Lett., vol. 18, no. 02 , p. $1940006,2019$.

[30] B. Hille, Ion Channels Of Excitable Membranes. Sunderland, MA: Sinauer Associates, 3rd ed., 2001.

[31] D. J. Aidley and P. R. Stanfield, Ion Channels: Molecules in Action. Cambridge: CUP, 2000.

[32] S.-H. Chung, V. Krishnamurthy, and O. S. Andersen, Biological Membrane Ion Channels Dynamics, Structure, and Ap- 
plications. Springer, 2007.

[33] A. Thompson, I. Kim, T. Panosian, T. Iverson, T. Allen, and C. Nimigean, "Mechanism of potassium-channel selectivity revealed by $\mathrm{Na}^{+}$and $\mathrm{Li}^{+}$binding sites within the KcsA pore," Nat. Struct. Mol. Biol., vol. 16, no. 12, p. 1317, 2009.

[34] C. Nimigean and T. Allen, "Origins of ion selectivity in potassium channels from the perspective of channel block," $J$. Gen. Physiol., vol. 137, no. 5, pp. 405-413, 2011.

[35] I. Kim and T. W. Allen, "On the selective ion binding hypothesis for potassium channels," Proc. Nat. Acad. Sci. (USA)., vol. 108, no. 44, pp. 17963-17968, 2011.

[36] C. N. Schutz and A. Warshel, "What are the dielectric "constants" of proteins and how to validate electrostatic models?," Proteins, vol. 44, no. 4, pp. 400-417, 2001.

[37] J. A. Ng, T. Vora, V. Krishnamurthy, and S.-H. Chung, "Estimating the dielectric constant of the channel protein and pore," Eur. Biophys. J., vol. 37, no. 2, pp. 213-222, 2008.

[38] D. Bucher, S. Raugei, L. Guidoni, M. Dal Peraro, U. Rothlisberger, P. Carloni, and M. L. Klein, "Polarization effects and charge transfer in the kcsa potassium channel," Biophys. Chem., vol. 124, no. 3, pp. 292-301, 2006.

[39] S. Kraszewski, C. Boiteux, C. Ramseyer, and C. Girardet, "Determination of the charge profile in the kcsa selectivity filter using ab initio calculations and molecular dynamics simulations," Phys. Chem. Chem. Phys., vol. 11, no. 38, pp. 8606-8613, 2009.

[40] C. Guardiani, P. Rodger, O. Fedorenko, S. Roberts, and I. Khovanov, "Sodium binding sites and permeation mechanism in the NaChBac channel: A molecular dynamics study," J. Chem. Theor. Comp., p. 10.1021/acs.jctc.6b01035, 2016.

[41] S. Furini, P. Barbini, and C. Domene, "Effects of the protonation state of the EEEE motif of a bacterial Na ${ }^{+}$-channel on conduction and pore structure," Biophys. J., vol. 106, pp. 2175-2183, 2014.

[42] B. Corry and M. Thomas, "Mechanism of ion permeation and selectivity in a voltage gated sodium channel," J. Am. Chem. Soc., vol. 134, no. 3, pp. 1840-1846, 2012.

[43] C. Boiteux, I. Vorobyov, and T. W. Allen, "Ion conduction and conformational flexibility of a bacterial voltage-gated sodium channel," Proc. Natl. Acad. Sci. U.S.A, vol. 111, no. 9, pp. 3454-3459, 2014.

[44] O. A. Fedorenko, I. K. Kaufman, W. A. Gibby, M. L. Barabash, D. G. Luchinsky, S. K. Roberts, and P. V. McClintock, "Ionic coulomb blockade and the determinants of selectivity in the nachbac bacterial sodium channel," Biochimica et Biophysica Acta (BBA)-Biomembranes, p. 183301, 2020.

[45] A. Damjanovic, A. Y. Chen, R. L. Rosenberg, D. R. Roe, X. Wu, and B. R. Brooks, "Protonation state of the selectivity filter of bacterial voltage-gated sodium channels is modulated by ions," Proteins: Structure, Function, and Bioinformatics, vol. 88 , no. 3, pp. 527-539, 2020 .

[46] K. M. Callahan and B. Roux, "Molecular dynamics of ion conduction through the selectivity filter of the navab sodium channel," J. Phys. Chem. B, vol. 122, no. 44, pp. 10126-10142, 2018.

[47] C. Tilegenova, D. M. Cortes, N. Jahovic, E. Hardy, P. Hariharan, L. Guan, and L. G. Cuello, "Structure, function, and ion-binding properties of $\mathrm{a} \mathrm{k}^{+}$channel stabilized in the 2,4-ion bound configuration," Proc. Nat. Acad. Sci. (USA), vol. 116, pp. 16829-16834, aug 2019.

[48] J. Morais-Cabral, Y. Zhou, and R. MacKinnon, "Energetic optimization of ion conduction rate by the $\mathrm{K}^{+}$selectivity filter," Nature, vol. 414, no. 6859, pp. 37-42, 2001.

[49] D. Köpfer, C. Song, T. Gruene, G. Sheldrick, U. Zachariae, and B. de Groot, "Ion permeation in $\mathrm{K}^{+}$channels occurs by direct Coulomb knock-on," Science, vol. 346, no. 6207, pp. 352-355, 2014.

[50] W. Kopec, D. A. Köpfer, O. N. Vickery, A. S. Bondarenko, T. L. Jansen, B. L. de Groot, and U. Zachariae, "Direct knock-on of desolvated ions governs strict ion selectivity in $\mathrm{K}^{+}$channels," Nat. Chem., vol. 10, no. 8, p. 813, 2018.

[51] Y. Zhou and R. MacKinnon, "The occupancy of ions in the $\mathrm{K}^{+}$selectivity filter: Charge balance and coupling of ion binding to a protein conformational change underlie high conduction rates," J. Mole. Biol., vol. 333, no. 5, pp. 965-975, 2003.

[52] C. M. Armstrong, "Packaging life: the origin of ion-selective channels," J. Biophys., vol. 109, no. 2, pp. 173-177, 2015.

[53] In this instance $\mathrm{T}=$ threonine, $\mathrm{V}=$ valine, $\mathrm{G}=$ glycine, $\mathrm{Y}=$ tyrosine and $\mathrm{x}$ indicates a different residue.

[54] D. Naranjo, H. Moldenhauer, M. Pincuntureo, and I. Díaz-Franulic, "Pore size matters for potassium channel conductance," Journal of General Physiology, vol. 148, no. 4, pp. 277-291, 2016.

[55] I. Díaz-Franulic, R. V. Sepúlveda, N. Navarro-Quezada, F. González-Nilo, and D. Naranjo, "Pore dimensions and the role of occupancy in unitary conductance of shaker k channels," Journal of General Physiology, vol. 146, no. 2, pp. 133-146, 2015.

[56] P. Nelson, "A permeation theory for single-file ion channels: One- and two-step models," J. Chem. Phys., vol. 134, pp. 165102-165114, 2011.

[57] S. Agah, M. Pasquali, and A. Kolomeisky, "Theoretical analysis of selectivity mechanisms in molecular transport through channels and nanopores," J. Chem. Phys., vol. 142, no. 4, p. 044705, 2015.

[58] B. Hille, "Ionic selectivity, saturation, and block in sodium channels. a four-barrier model.," J. Gen. Phys., vol. 66, no. 5, pp. 535-560, 1975.

[59] B. Eisenberg, "Crowded charges in ion channels," in Adv. Chem. Phys. (S. A. Rice and A. R. Dinner, eds.), vol. 148 of Advances in Chemical Physics, pp. 77-223, Malden, MA: Wiley-Blackwell, 2012.

[60] P. Hänggi, P. Talkner, and M. Borkovec, "Reaction-rate theory: fifty years after Kramers," Rev. Mod. Phys., vol. 62, no. 2, p. 251, 1990.

[61] W. A. T. Gibby, M. L. Barabash, C. Guardiani, D. G. Luchinsky, O. A. Fedorenko, S. K. Roberts, and P. V. E. McClintock, "Theory and experiments on multi-ion permeation and selectivity in the nachbac ion channel," Fluct. Noise Lett., vol. 18, no. 02, p. 1940007, 2019. 
[62] W. D. Cornell, P. Cieplak, C. I. Bayly, I. R. Gould, K. M. Merz, D. M. Ferguson, D. C. Spellmeyer, T. Fox, J. W. Caldwell, and P. A. Kollman, "A second generation force field for the simulation of proteins, nucleic acids, and organic molecules," Journal of the American Chemical Society, vol. 117, no. 19, pp. 5179-5197, 1995.

[63] B. R. Brooks, R. E. Bruccoleri, B. D. Olafson, D. J. States, S. a. Swaminathan, and M. Karplus, "Charmm: a program for macromolecular energy, minimization, and dynamics calculations," Journal of computational chemistry, vol. 4, no. 2, pp. 187-217, 1983.

[64] A. D. MacKerell Jr, B. Brooks, C. L. Brooks III, L. Nilsson, B. Roux, Y. Won, and M. Karplus, "Charmm: the energy function and its parameterization," Encyclopedia of computational chemistry, vol. 1, 2002.

[65] B. R. Brooks, C. L. Brooks III, A. D. Mackerell Jr, L. Nilsson, R. J. Petrella, B. Roux, Y. Won, G. Archontis, C. Bartels, S. Boresch, et al., "Charmm: the biomolecular simulation program," Journal of computational chemistry, vol. 30, no. 10, pp. 1545-1614, 2009.

[66] J. A. Lemkul, J. Huang, B. Roux, and A. D. MacKerell Jr, "An empirical polarizable force field based on the classical drude oscillator model: development history and recent applications," Chemical reviews, vol. 116, no. 9, pp. 4983-5013, 2016.

[67] V. Ngo, H. Li, A. D. MacKerell Jr, T. W. Allen, B. Roux, and S. Noskov, "Polarization effects in water-mediated selective cation transport across a narrow transmembrane channel," Journal of Chemical Theory and Computation, 2021.

[68] W. A. T. Gibby, C. M. L. Barabash, Guardiani, D. G., Luchinsky, and P. V. E. McClintock, "The role of noise in determining selective ionic conduction through nano-pores," in 2018 IEEE 13th Nanotechnology Materials and Devices Conference (NMDC), pp. 1-6, IEEE, 2018.

[69] M. Barabash, W. Gibby, C. Guardiani, A. Smolyanitsky, D. Luchinsky, and P. McClintock, "Origin and control of ionic hydration patterns in nanopores," Researchsquare, 2020.

[70] G. Lamoureux, A. D. MacKerell Jr, and B. Roux, "A simple polarizable model of water based on classical drude oscillators," The Journal of chemical physics, vol. 119, no. 10, pp. 5185-5197, 2003.

[71] G. Lamoureux and B. Roux, "Modeling induced polarization with classical drude oscillators: Theory and molecular dynamics simulation algorithm," The Journal of chemical physics, vol. 119, no. 6, pp. 3025-3039, 2003.

[72] J. Feng, K. Liu, M. Graf, D. Dumcenco, A. Kis, M. Di Ventra, and A. Radenovic, "Observation of ionic Coulomb blockade in nanopores," Nat. Mater., vol. 15, no. 8, pp. $850-855,2016$.

[73] N. Kavokine, S. Marbach, A. Siria, and L. Bocquet, "Ionic coulomb blockade as a fractional wien effect," Nat. Nanotechnol., vol. 14, pp. 573-578, 2019.

[74] B. Roux, "Statistical mechanical equilibrium theory of selective ion channels," Biophys. J., vol. 77, no. 1, pp. 139-153, 1999.

[75] W. Im, S. Seefeld, and B. Roux, "A grand canonical Monte Carlo Brownian dynamics algorithm for simulating ion channels," Biophys. J., vol. 79, no. 2, pp. 788-801, 2000.

[76] Y. Marcus, "Thermodynamics of solvation of ions. part 5.-gibbs free energy of hydration at 298.15 k," J. Chem. Soc. Faraday Trans., vol. 87, no. 18, pp. 2995-2999, 1991.

[77] T. Baştuğ and S. Kuyucak, "Comparative study of the energetics of ion permeation in kv1. 2 and kcsa potassium channels," Biophysical journal, vol. 100, no. 3, pp. 629-636, 2011.

[78] P. Dixit, M. Merchant, and D. Asthagiri, "Ion selectivity in the KcsA potassium channel from the perspective of the ion binding site," Biophys. J., vol. 96, pp. 2138-2145, jan 2009.

[79] S. Noskov and B. Roux, "Importance of hydration and dynamics on the selectivity of the KcsA and NaK channels," J. Gen. Physiol., vol. 129, no. 2, pp. 135-143, 2007.

[80] B. Egwolf and B. Roux, "Ion selectivity of the kcsa channel: a perspective from multi-ion free energy landscapes," J. Mol. Biol., vol. 401, no. 5, pp. 831-842, 2010.

[81] I. Kim and T. W. Allen, "On the selective ion binding hypothesis for potassium channels," Proc. Nat. Acad. Sci. (USA), vol. 108 , no. 44, pp. 17963-17968, 2011.

[82] In this instance $\mathrm{T}=$ threonine, $\mathrm{V}=$ valine, $\mathrm{G}=$ glycine, $\mathrm{Y}=$ tyrosine and $\mathrm{x}$ indicates a different residue. 

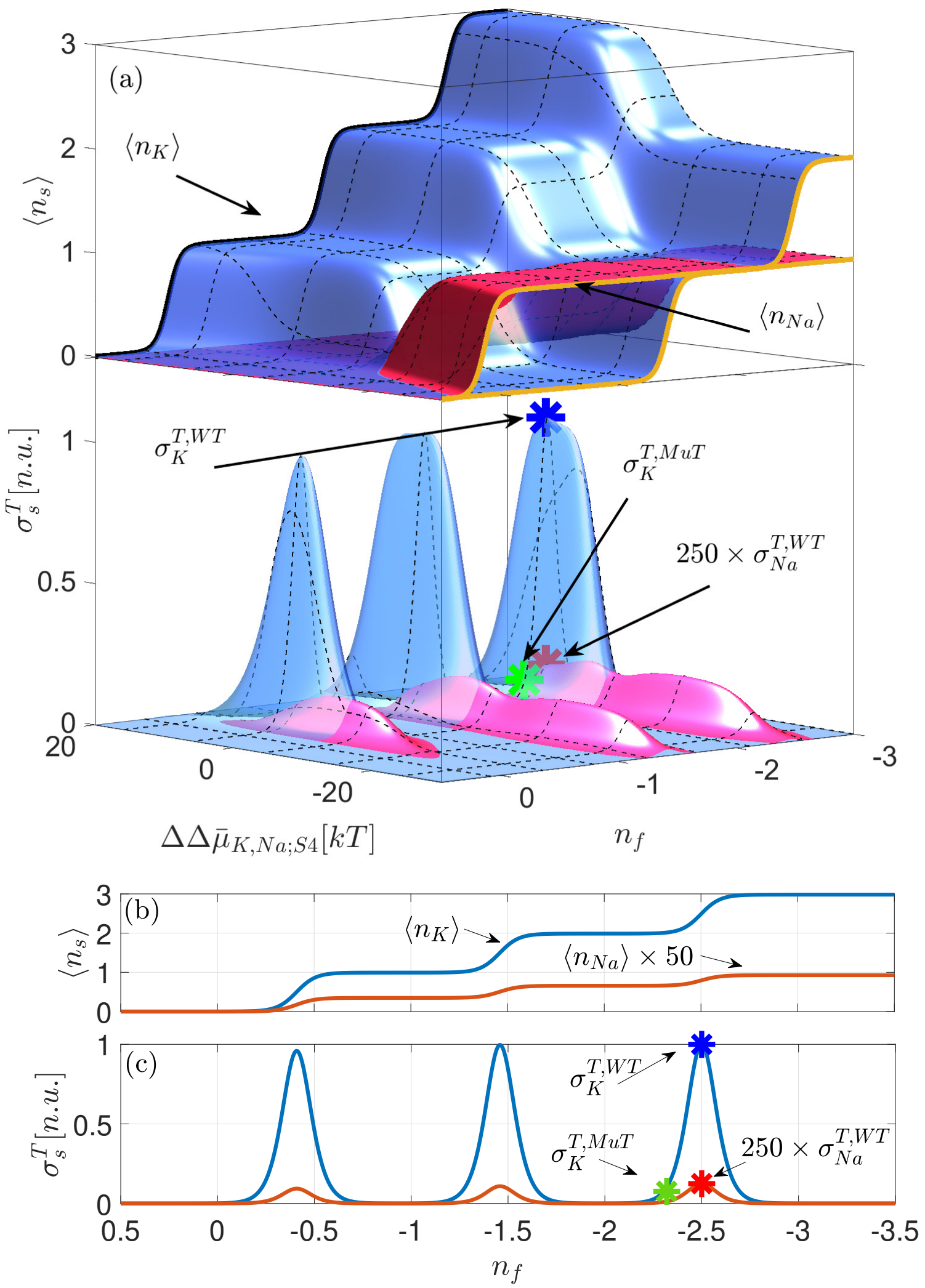

FIG. 5. (a) $\mathrm{K}^{+}$(blue) and $\mathrm{Na}^{+}$(red) occupancy (top) and conductivity (bottom) vs. $n_{f}$ and $\Delta \bar{\mu}_{K, N a ; S 4}$ in symmetrical $0.2 \mathrm{M}$ mixed bulk solutions. Conductivity and occupancy form a set of resonant peaks and steps respectively. Each step maximises under the conditions of barrier-less knock on, it being the favoured species and minimal difference in site affinity. Using identical parameters to Fig. 2. in the main manuscript obtained through experimental comparison, we indicate the $\mathrm{WT} \mathrm{K}^{+}$(blue) and $\mathrm{Na}^{+}$(red) and $\mathrm{MuT} \mathrm{K}{ }^{+}$(green) conductivity's via coloured stars. Selectivity appears via the shift in both occupancy and conductivity from $\mathrm{K}^{+}$(blue) to $\mathrm{Na}^{+}$(red) surfaces, and the conductivity ratio yields $\sigma_{K}^{T, W T} / \sigma_{N a}^{T, W T} \sim 2 \times 10^{3}$. Note this corresponds to Fig. 3. of the main text. (b) and (c) are 2-D cuts of this figure with $\Delta \mu_{K, S 4}=6.2 k T \Delta \mu_{N a, S 4}=0.2 k T$. Note that the green star has been added onto this figure and is calculated with $\Delta \mu_{K, S 4}=5.2 k T$. As a result of this change in affinity and recalling that the S4 site geometry varied the green star is off the main curve for the WT, but serves as a guide to the reader. 


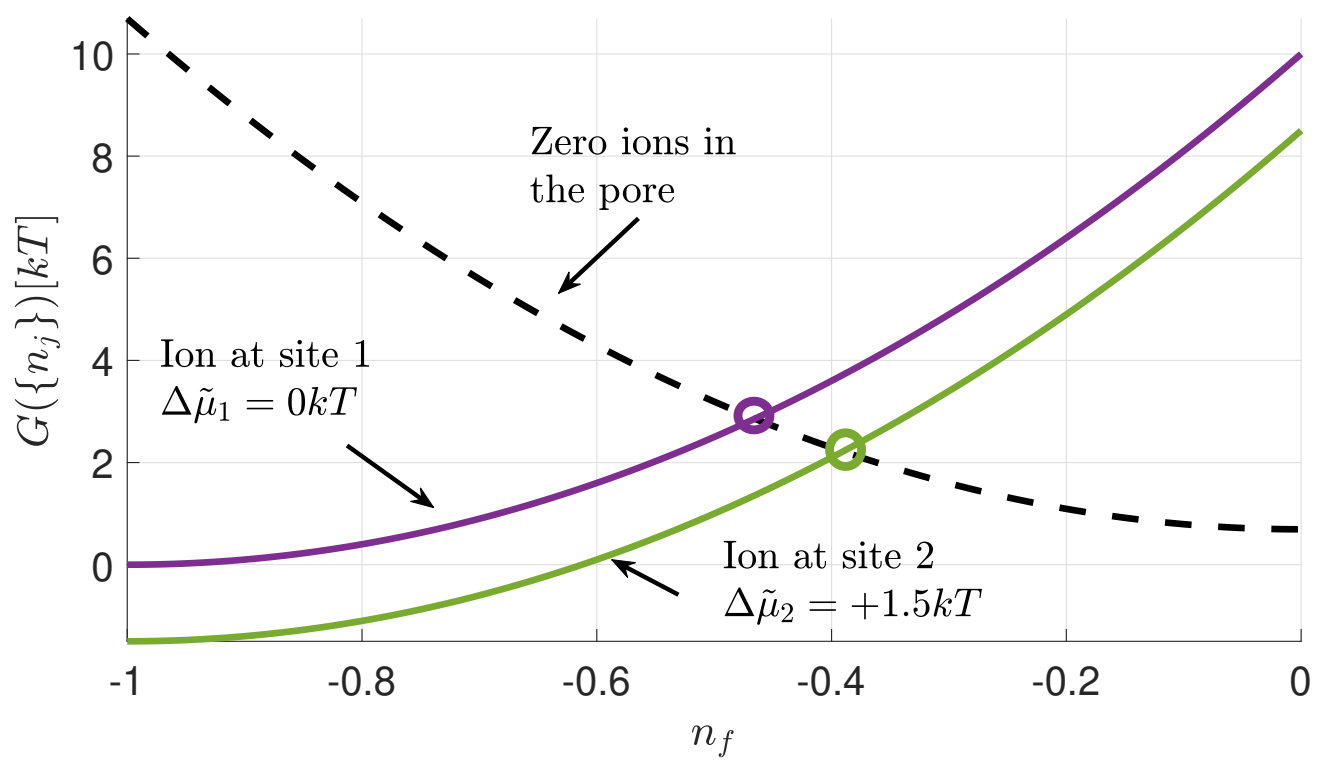

FIG. 6. Free energy $G$ vs. the fixed charge $n_{f}$. The black dashed curve represents the state without ions; the purple and green curves represent states with an ion at site 1 or 2 respectively. The purple and green circles highlight the degeneracy knock-on condition between states with 1 and 0 ions (see Eqn. (46)).
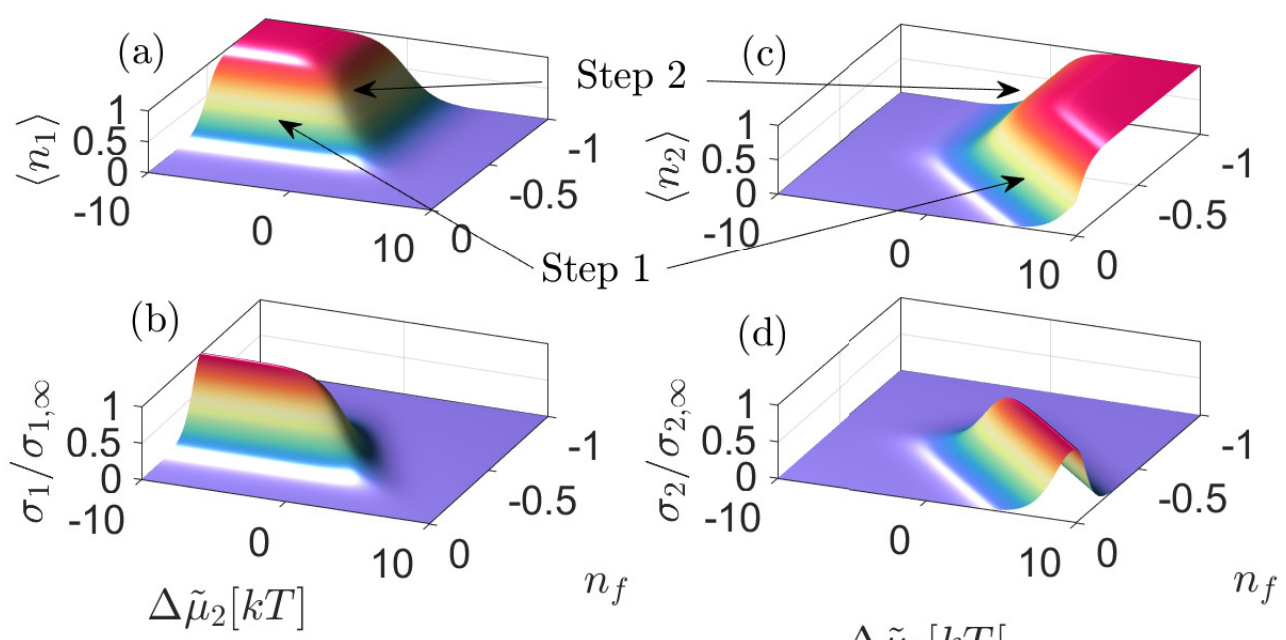

$\Delta \tilde{\mu}_{2}[k T[$

FIG. 7. Occupancy (a,c) and normalised conductivity (b,d) into site 1 and 2 respectively, are plotted $v s . n_{f}$ and $\Delta \bar{\mu}_{2}$. Two steps in occupancy form, one when an ion is entering the pore i.e. facing the $\Delta \bar{\mu}_{2}$ axis and the second when an ion is in the pore but is either in site 1 or 2 . The former step corresponds to the peak in conductivity into the site. 

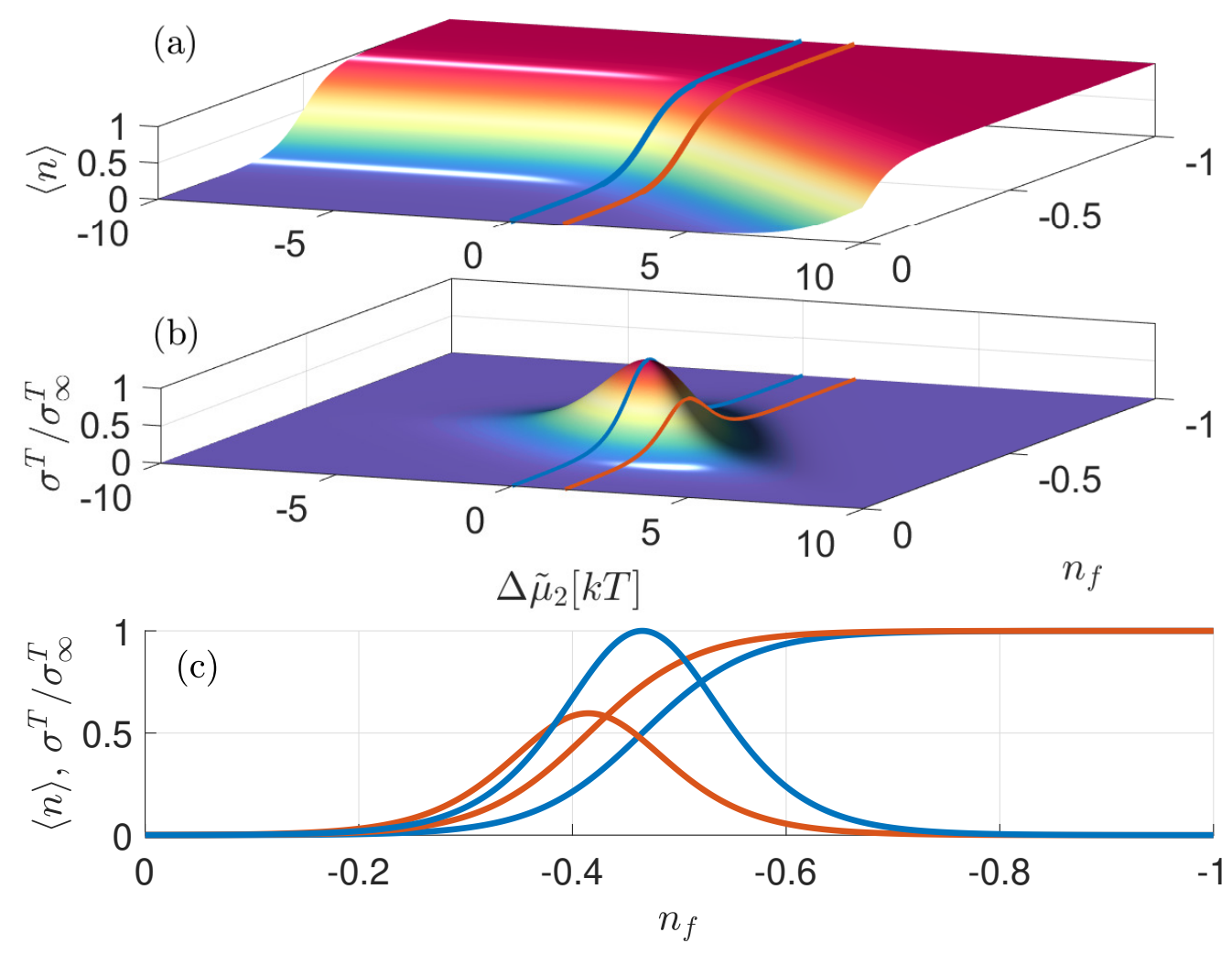

FIG. 8. Occupancy (a) and normalised conductivity (b) plotted $v s . n_{f}$ and $\Delta \tilde{\mu}_{2}$. Occupancy forms a step that varies dependent on $n_{f}$ and $\Delta \tilde{\mu}_{2}$ only when site 2 is more favoured. The midpoint of this step corresponds to the degeneracy between states given by Eqn. (46). Conductivity forms a peak around this point if the two binding sites also share affinity i.e. $\left|\Delta \tilde{\mu}_{1}-\Delta \tilde{\mu}_{2}\right| \sim 0$. In analogy with quantum transport through quantum dots the phenomena along the $n_{f}$ axis can be attributed to ionic Coulomb blockade.
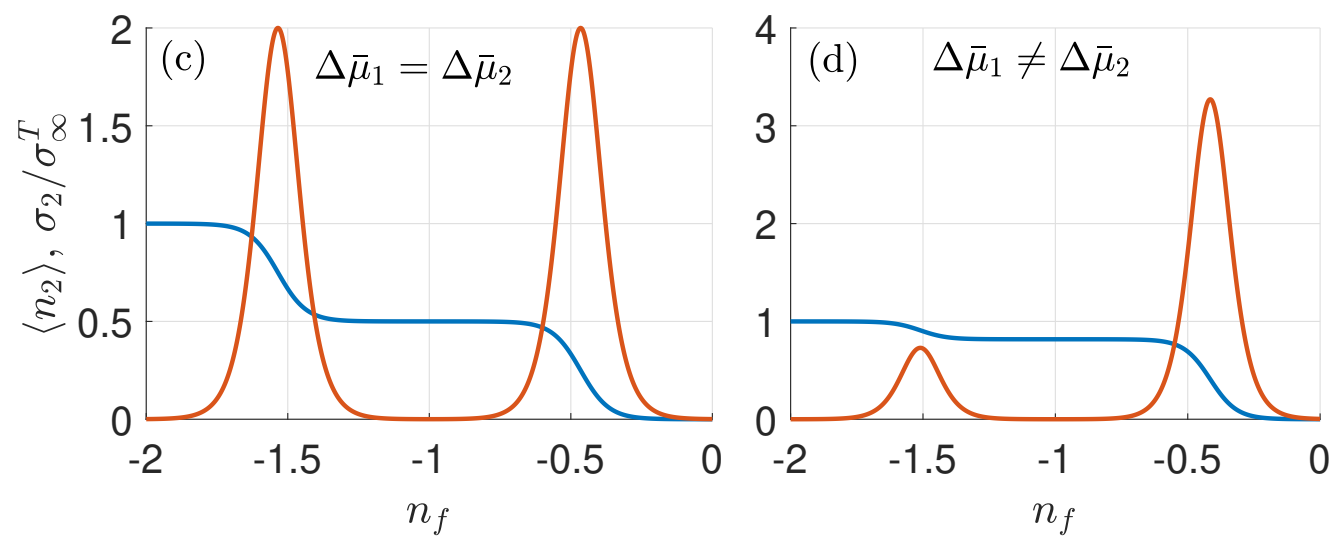

FIG. 9. (a) Energy spectra vs. $n_{f}$. The purple and green curves represent the single-ion states with occupation at sites 1 or 2 , respectively; the red and black curves represent the dual-ion and zero-ion states. Coloured circles highlight the degeneracy condition. 

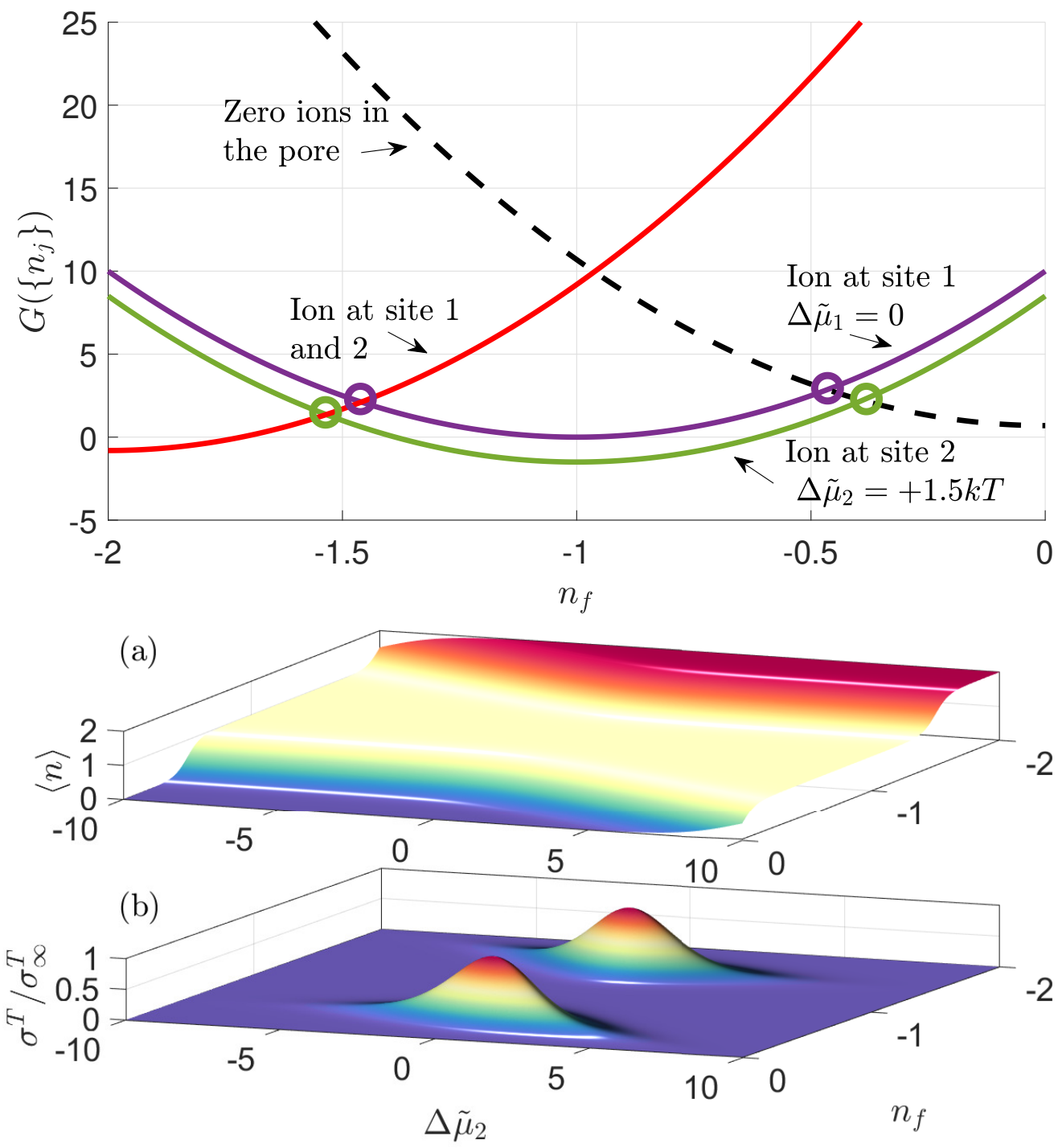

FIG. 10. Occupancy (a), normalised conductivity (b) traces when the sites share affinity (c) and there is a favoured site (d). Each conductivity peak again forms close to the degeneracy condition or mid-point of occupancy step, and the frequency between peaks can be calculated from the difference between energy levels. Both functions share an anti-symmetry along the $\Delta \bar{\mu}_{2}$ plane because, if either site is full, a transition is only possible to the other one. When sites share affinity the total conductivity involves transitions at both sites and so each site shares conductivity resulting in a smaller total. If an energy difference exists between sites then conductivity into each site is only possible provided that the site is empty. As a result in (d) conductivity is significantly reduced in the 1-2 transition because it is almost filled. If it was filled then the conductivity would be negligible. 


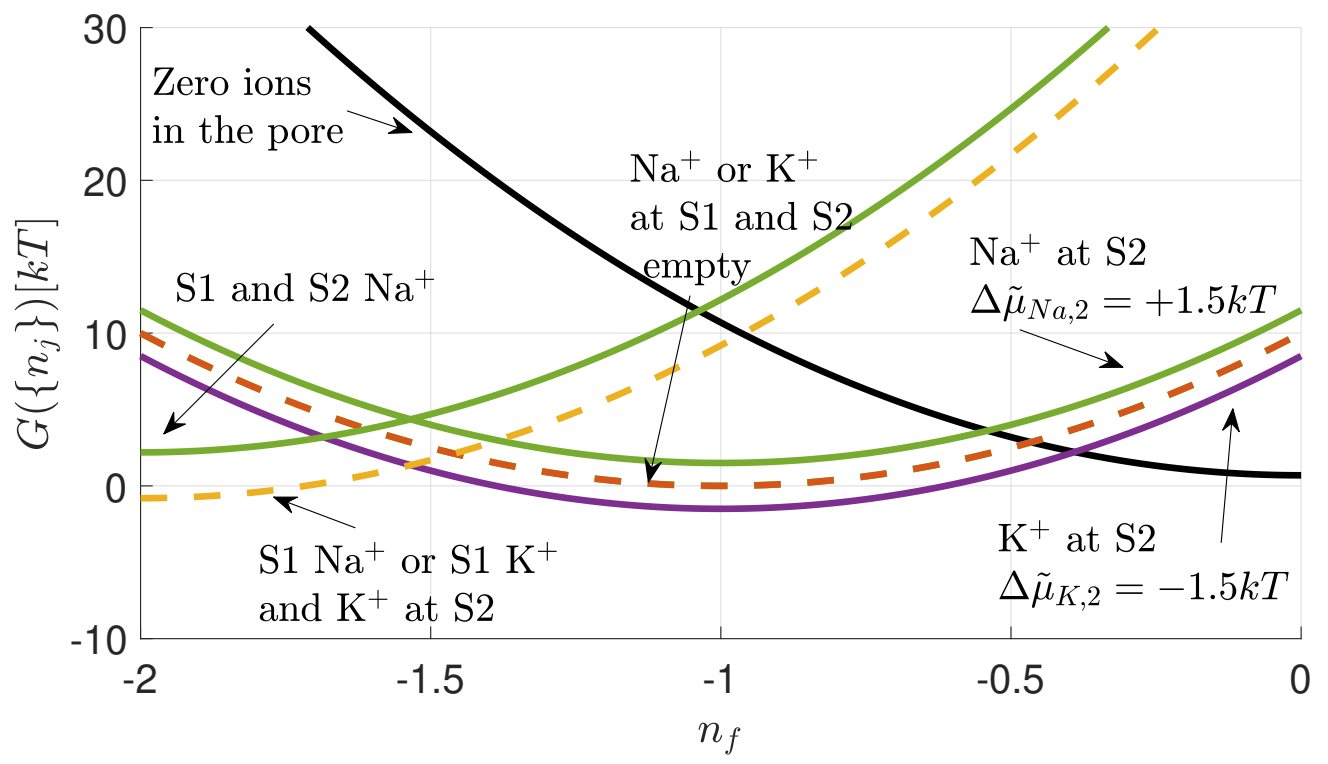

FIG. 11. Energy Spectra vs. $n_{f}$. The solid purple and green curves correspond to pure $\mathrm{K}^{+}$and $\mathrm{Na}^{+}$states, the dashed orange represents either $\mathrm{K}^{+}$or $\mathrm{Na}^{+}$at site 1 , the dashed yellow line is either $\mathrm{K}^{+}$or $\mathrm{Na}^{+}$at site 1 and $\mathrm{K}^{+}$at site 2 , and finally the black line denotes the zero-ion state.
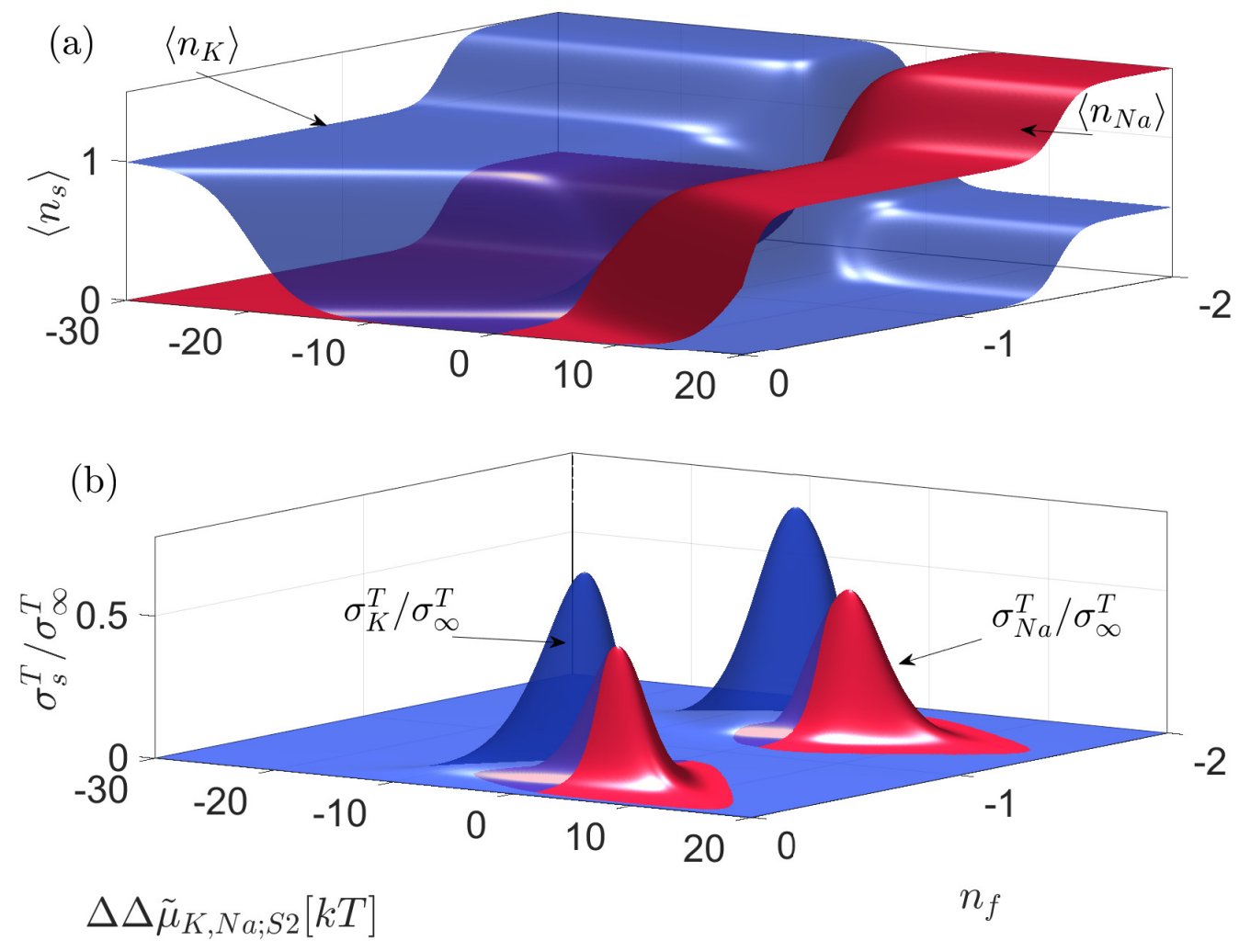

FIG. 12. $\mathrm{K}^{+}$(blue) and $\mathrm{Na}^{+}$(red) occupancy (a), and normalised conductivity (b) plotted vs. $n_{f}$ and $\Delta \Delta \bar{\mu}_{K, N a ; 2}$. 